\title{
Les habitants des ruines
}

Comme je l'ai évoqué précédemment, l'espace des ruines tel que décrit dans le corpus prophétique est l'un des contextes majeurs où des démons apparaissent dans la LXX. Toutefois, la présence de ces derniers dans la Bible hébraïque n'est pas, pour sa part, si explicite et demeure sujette à débat. Les rapports entre l'hébreu et le grec doivent donc être étudiés afin de mieux situer ces textes dans leurs relations réciproques et de comprendre les trajectoires qui ont mené à une émergence du démoniaque en ce contexte. À ma connaissance, ce type d'analyse n'a encore jamais été réalisé1 et mérite donc d'être tenté.

Les oracles qu'on appelle d'habitude «contre les nations » évoquent fréquemment des paysages de villes en ruines, un motif qui annonce la défaite future des ennemis d'Israël. Il est possible d'identifier ce topos par une série d'éléments récurrents. En effet dans ces passages, la destruction des villes n'est autre que la conséquence de la colère de Yhwh et cet anéantissement signifie métonymiquement la perte du territoire entier. L'espace urbain et les champs cultivés sont abandonnés par les hommes et les animaux domestiques et les vestiges des cités se remplissent alors de végétation sauvage et sont repeuplés par toutes sortes d'animaux et de créatures. Les tannîm, qui désignent probablement des canidés du désert ${ }^{2}$, semblent être des marqueurs de ce territoire hostile à la civilisation, comme l'annonce l'expression məôn tannîm, « repaire des tannîm», utilisée plusieurs fois en Jérémie pour désigner des ruines. L'oracle contre le territoire de Ḥașor en Jer 49,33 en donne un exemple ${ }^{3}$ :

1 Pour une analyse préliminaire menée conjointement par Christophe Nihan et moi-même, voir Angelini 2017 ; Nihan 2017.

2 L'hébreu connaît au moins trois termes qui désignent des canidés sauvages : tannîm, 'iyyîm, les deux attestés exclusivement à la forme plurielle, et šû́ăl, traditionnellement traduit par «renard », qui apparaît parfois comme animal des ruines (Lam 5,18; Ez 13,4). Les deux derniers zoonymes ont un certain nombre de parallèles dans d'autres langues sémitiques (voir respectivement SED II, no. 21 et 237 ; et infra, p. 157), alors que *tan est isolé et d'étymologie difficile. Nous ne sommes pas en mesure d'établir précisément l'espèce à laquelle le mot tannîm se réfère et il n'est pas possible de trancher entre « chacal » et « hyène » : voir, à ce sujet, Frevel 1995 .

3 Ḥașor n'indique pas ici la ville du nord de la Palestine, mais un territoire au sud de la Palestine peuplé par des tribus arabes, qui, ici et en Jer 49,28-30, est couplé avec Kedar (Lundbom 2004, p. 353). Pour l'expression « repaire des tannîm», voir également Jer 9,10 (Jérusalem);

(C) ANNA ANGELINI, 2021 | DOI:10.1163/9789004468474_007

This is an open access chapter distributed under the terms of the CC BY-NC-ND 4.o licensenge 


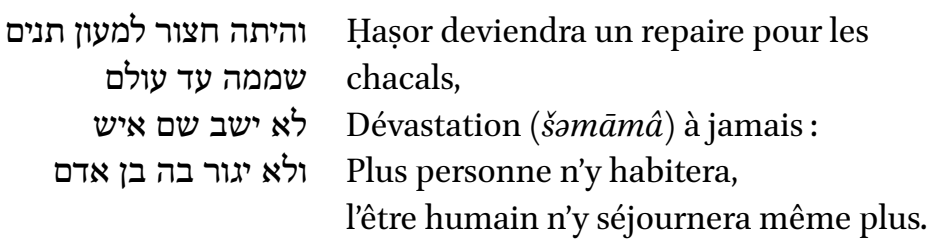

Le motif, partagé par plusieurs recueils prophétiques, est pour sa part une variation du thème beaucoup plus répandu des terres réduites «à la dévastation » : ce thème est signalé par une série de mots-clés construits sur la racine šmm, « être détruit », « être vide », mais également « être choqué », « horrifié » (šammâ, məšammâ, šəmāmâ). Il est particulièrement aimé par Jérémie qui l'applique notamment à Jérusalem et aux villes de Juda ${ }^{4}$ :

9 Sur les montagnes s'élèvent mes pleurs et mon deuil, et sur les habitations du désert mon chant funéraire.

Car elles sont incendiées, sans que personne n'y passe, et on n'entend plus le bruit des troupeaux.

Des oiseaux du ciel aux quadrupèdes, (tous) se sont enfuis.

${ }^{10}$ Je fais de Jérusalem un tas de pierres, un repaire de chacals, et des villes de Juda, des lieux dévastés (šəmāmâ), vidés de leurs habitants.

11 [...] Pourquoi le pays est-il ruiné, brûlé comme le désert où personne ne passe?

Un destin similaire est également prévu pour les ennemis d'Israël, dont notamment Edom ${ }^{5}$ :

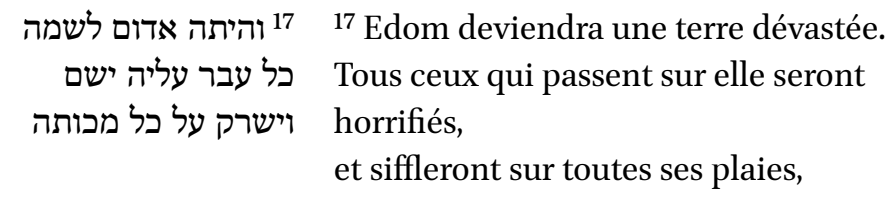

\footnotetext{
10,22 (Juda); 51,37 (Babylone). Comparer, en outre, avec Ps 44,20; Nah 2,12 («repaire du lion »). Is 34,13 et 35,7 ont l'expression nawê tannîm, «la résidence des tannîm». Pour une
partie des passages traités ici en hébreu je reprends les traductions de Christophe Nihan lion »). Is 34,13 et 35,7 ont l'expression nawê tannîm, «la résidence des tannîm». Pour une
partie des passages traités ici en hébreu je reprends les traductions de Christophe Nihan (2017, p. 113-115), avec quelques modifications. Les autres traductions sont miennes.

4 Jer 9,9-11; voir aussi sur Juda Jer 10,22. 5 Jer 49,17-18.
} 


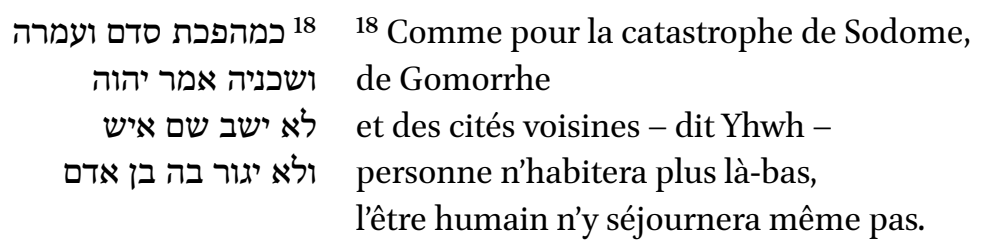

En outre, la ruine est le leitmotiv du long oracle sur le jugement de Babylone qui occupe les derniers chapitres du recueil $\left(5^{\mathrm{O}-51}\right)$, comme le montre déjà l'ouverture $(5 \circ, 3)$ :

${ }^{3}$ Car contre elle (= Babylone) monte une nation du nord, qui transforme son pays en dévastation (ləšammâ), personne n'y résidera :

ni hommes ni bêtes, (tous) se sont enfuis.

Le motif des terres changées en étendues désolées et vidées de toute présence humaine est également présent en Isaïe, avec une référence accrue à Babylone et à Edom ${ }^{6}$. La menace peut être, à l'occasion, proférée aussi contre Juda, comme par exemple en Is $32,12-14$ :

${ }^{12}$ En se frappant la poitrine ils gémissent sur les belles campagnes riantes, sur les vignes fécondes, ${ }^{13}$ sur la terre de mon peuple des buissons d'épines montent, oui, sur toutes les maisons joyeuses de la ville exultante.

${ }^{14}$ Le palais est abandonné, la ville tumultueuse est délaissée.

La colline et la tour de garde deviendront des cavernes pour toujours, joie des ânes sauvages, pâturage des troupeaux.

Enfin, quelques références sont connues parmi les douze petits prophètes. Toutefois, dans ces cas, on ne trouve que des mentions assez brèves des villes qui seront réduites à létat de ruines, sans qu'il soit fait allusion à l'arrivée d'animaux sauvages sur le territoire ${ }^{7}$. Une exception remarquable est constituée par l'oracle contre Ninive en Soph 2,13-15, qui décrit l'invasion par des rapaces de vestiges de palais luxueux:

6 Is 23,$13 ; 13,19-22 ; 14,23 ; 34,11-17$.

7 Par exemple en Am 4,11 et Mich 1,6 (Samarie) ; 3,12 (Jérusalem); Soph 2,9 (Moab et Ammon). 


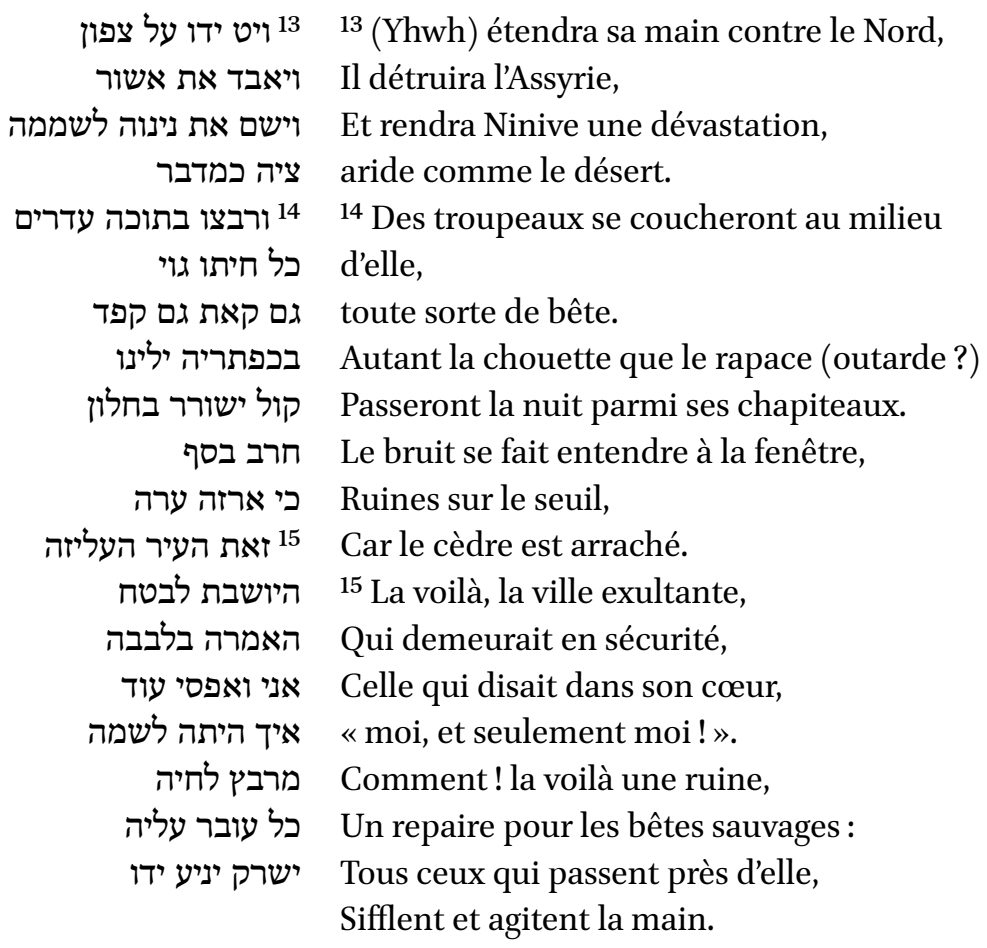

Parmi les différents oracles qui utilisent ce topos, certains présentent un intérêt particulier car ils partagent et combinent plusieurs éléments qui ne se trouvent pas réunis dans les autres passages. Premièrement, la description du paysage des ruines y est particulièrement développée et elle inclut la mention d'animaux et de créatures qui viennent peupler cet espace. Deuxièmement, la disparition des villes est implicitement ou explicitement comparée à celle de Sodome et Gomorrhe, ce qui souligne le caractère irrémédiable et définitif de la destruction à laquelle le territoire est destiné. Troisièmement, le motif des ruines occupe une position stratégique à l'intérieur des collections respectives, en servant de clôture à des sections entières d'oracles de malheur. Il s'agit donc d'un topos qui vient renforcer l'efficacité du jugement proféré. C'est le cas de l'oracle contre Ninive en Soph 2 ainsi que de la chute de Babylone annoncée en Jer 50,39-40 :

39 לכן ישבו ציים את איים וישבו בה בנות יענה ולא תשב עוד לנצח בעות ויער ולא תשכון עד דור ודור
${ }^{39}$ C'est pourquoi les créatures du désert y habiteront avec les hurleurs, et les autruches y habiteront également ; Babylone ne sera plus jamais habitée, de génération en génération on n'y résidera pas. 


\begin{tabular}{|c|c|}
\hline 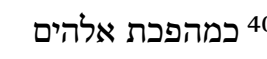 & ${ }^{40}$ Ce sera comme pour la catastrophe divine \\
\hline 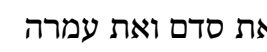 & qui a frappé Sodome, Gomorrhe, \\
\hline את שכניה נאם יהוה & $\begin{array}{l}\text { et les cités voisines - oracle de Yhwh. } \\
\text { personne n'habitera plus là-bas, }\end{array}$ \\
\hline לא יגור בה בן אדם & l'être humain n'y séjournera même pas. \\
\hline
\end{tabular}

Cette formulation est très similaire à celle que nous retrouvons dans le texte massorétique de Jer 51,37, qui est le dernier oracle présent dans le livre de Jérémie :

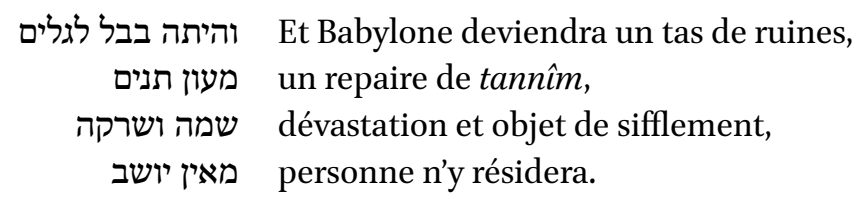

L'emplacement des oracles en Is 13 et 34, respectivement contre Babylone et contre Edom, mérite également d'être remarqué car le chapitre 13 (versets 19-22) conclut l'annonce de la destruction de Babylone et cette annonce ouvre, à son tour, la collection d'oracles contre les nations du premier Isaïe :

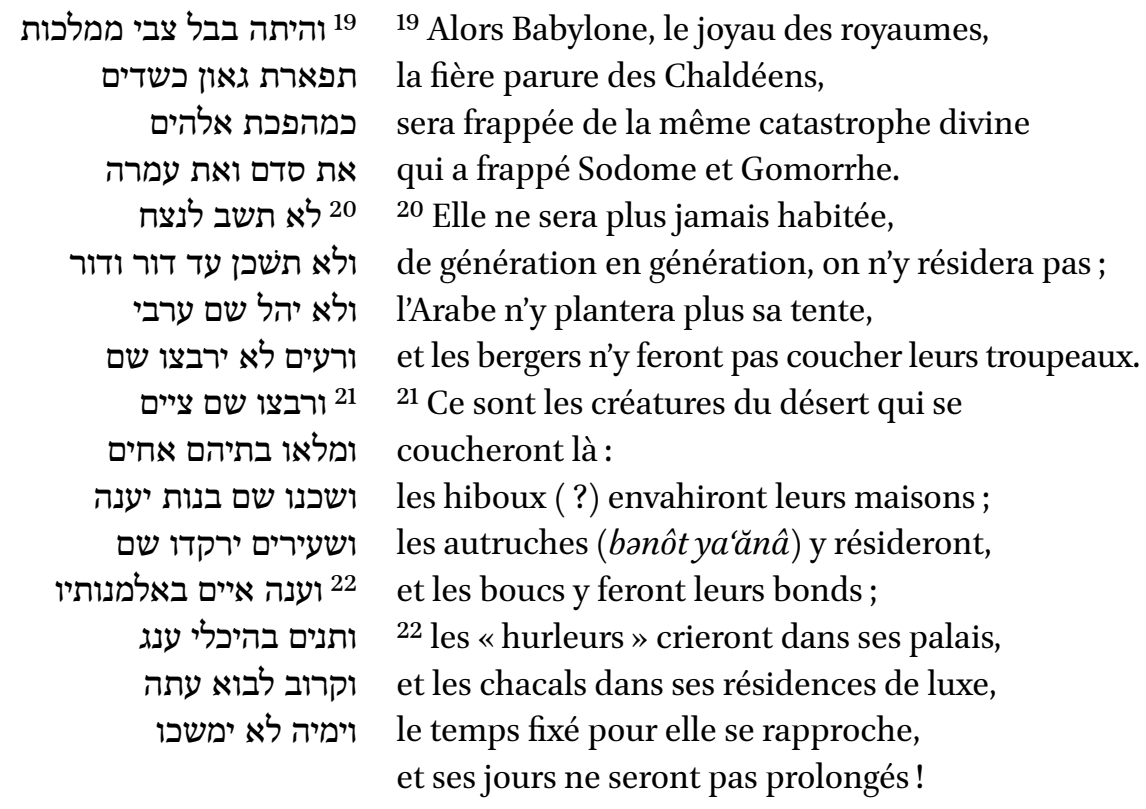


Quant au chapitre 34 (versets 11-17), il reprend directement le langage d'Is 13 et conclut les oracles de malheur du premier Isaïe, tout en préparant l'annonce de salut pour Sion :

9 ונהפכו נחליה לזפת 9 ועפרה לגפרית והיתה ארצה לזפת בערה לית ועפרה 10 לילה ויומם לא תכבה ליפת בערה לעולם יעלה עשנה מדור לדור תחרב עשל יער לנצח נצחים אין עבר בה מרור תור 11

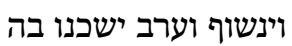

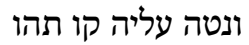
ואבני בהו 12

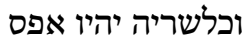
13 ועלתה ארמנתיה סירים קמוש וחוח במבצריה

והיתה נוה תנים

חציר לבנות יענה תנים

14 ושעיר על רעהו יקרא אך שם הרגיעה לילית ומצאה לה מנוח הריעת לילי 15 שמה קננה קפוז ותמלט מנוח

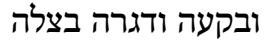

אך שם נקבצו דיות ובערה אשה רעותה 16 דרשו מעל ספר יהוה וקראו אחת מהנה לא נעדרה ספרה יהוה אשה רעותה לא פקדו לאורו

כיפי הוא צוה רעות ורוחו הוא קבצן
${ }^{9}$ Ses torrents (d'Edom) se changeront en poix, et sa poussière en soufre ; sa terre deviendra de la poix brûlante, ${ }^{10}$ de nuit comme de jour, elle ne s'éteindra pas : sa fumée s'élèvera à jamais ; de génération en génération (Edom) restera dévastée, plus jamais personne n'y passera.

${ }^{11}$ La chouette et le hibou ( ?) y habiteront; l'effraie et le corbeau y résideront. (Yhwh) étendra sur elle le cordeau du vide et la pierre (à mesurer) le chaos. ${ }^{12}$ Ses nobles n'y proclameront plus de roi, et tous ses princes auront disparu. ${ }^{13}$ Les ronces pousseront dans ses palais, les orties et les chardons dans ses tours; ce sera le repaire des chacals, le pâturage des bənôt yaănâ.

${ }^{14}$ Les créatures du désert et les hurleurs s'y rencontreront, chaque bouc y appellera son compagnon ;

c'est encore là que résidera Lilith, et qu'elle trouvera son lieu de repos.

${ }^{15}$ Le grand-duc ( ?) y fera son nid, pondra ses œufs, les couvera, et rassemblera (ses petits) sous son ombre; là encore, se rassembleront les vautours, chaque femelle avec son compagnon. ${ }^{16}$ Cherchez dans le livre de Yhwh, et lisez ! Aucune de ces créatures ne sera absente, Aucune n'aura à s'enquérir de son compagnon! Car c'est la bouche de Yhwh ${ }^{8}$ qui l'ordonne, c'est son esprit qui les rassemble ;

8 Je lis ici le tétragramme au lieu du pronom qui n'est pas pertinent du point de vue syntaxique. 


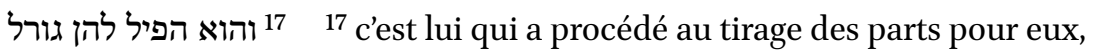
et c'est sa main qui leur a partagé le pays au cordeau ; c'est pour toujours qu'elles en prendront possession, de génération en génération elles y résideront.

Les trois critères ici définis (peuplement des ruines par plusieurs créatures sauvages, nature irrémédiable de la destruction et position stratégique à l'intérieur des collections prophétiques) permettent de délimiter un corpus cohérent sur lequel je vais focaliser mon attention. Il importe de relever que dans cette forme plus élaborée et complexe, les oracles ne sont jamais adressés à Jérusalem mais exclusivement aux nations. Puisque ces passages présentent des similarités de langage importantes, il faut commencer par s'interroger sur l'origine dudit motif des habitants des ruines, ainsi que sur les relations entre ces textes.

\subsection{Origines du motif et relations intertextuelles}

L'idée que l'espace des villes vouées à la destruction soit envahi par des animaux sauvages n'est pas propre à la Bible hébraïque ; on en trouve des parallèles très anciens au Proche Orient. Cette idée apparait, en effet, dans des typologies de textes divers, notamment dans des lamentations et des malédictions, et ce, dès l'époque paléo-babylonienne ${ }^{9}$. Un des poèmes de type Eršemma, à savoir un genre de lamentations sur des villes abandonnées à cause d'une colère divine, contient une plainte adressée au dieu Enlil à propos d'une série de villes détruites et laissées à la merci «des chiens, des loups et des démons »: ici le démon lil- est nommé à côté des canidés qui vagabondent dans les palais de la cité $^{10}$. Un autre exemple est représenté par la malédiction d'Agadé, un fameux texte mythologique d'origine sumérienne sur la chute d'Akkad, qui se termine par une malédiction lancée de la part des dieux contre la ville d'Akkad ${ }^{11}$ :

9 Les parallèles entre les passages prophétiques (surtout en Isaïe) et les textes procheorientaux qui font mention des ruines ont été étudiés par Hillers 1964, p. 44-64 et surtout par García Recio 1995 .

10 Le texte sumérien est préservé par une copie paléo-babylonienne et par une copie du premier millénaire : textes avec traduction anglaise chez Cohen 1981, p. 110-117, no. 1.1 et 1.2, respectivement lignes $32-34$ et $48-5$ o. Je remercie vivement Vérène Chalendar pour son aide à l'identification des animaux mentionnés dans ce passage.

11 Traduction de Pascal Attinger 2007, lignes 255-259: https://cdli.ucla.edu/dl/pdf/P469679 .pdf. Voir également Cooper, 1983, p. 62-63, 256 ; Pomponio 199o, p. 83-87. 
Puissent les «méchants» de la steppe silencieuse ne cesser de hurler! Sur ton kiuzga où avaient lieu des purifications, puisse le renard hantant les collines des ruines y trainer la queue, sur tes portes de ville établies dans le pays, puisse l'oiseau-sommeil, l'oiseau du cœur chagrin, y faire son nid!

Ce passage montre des similarités frappantes avec les textes d'Isaïe, notamment à cause du fait que, parmi les habitants de la ville abandonnée et réduite à l'état de steppe, sont mentionnés à la fois des renards, des oiseaux rapaces (l'oiseau-sommeil/oiseau du chagrin étant des hiboux) et des démons, désignés ici comme «les méchants de la steppe».

Le motif des habitants des ruines est, en outre, repris par les malédictions traditionnellement situées en conclusion des traités d'alliance afin d'empêcher que celle-ci ne soit rompue. L'attestation la plus importante du Levant, et la plus souvent citée, provient d'une des inscriptions araméennes de Sfiré ${ }^{12}$. La première plaque, aujourd'hui conservée au Musée de Damas, reproduit l'accord entre Mati'el, roi du petit royaume d'Arpad, et un autre gouverneur, dit Bar Ga'yah (dont l'identité est l'un des problèmes les plus discutés de la stèle). En cas de rupture du traité par Mati'el, toutes sortes de malédictions sont annoncées pour le royaume d'Arpad, telles que la destruction de la végétation et l'épuisement de la terre. En outre, une série de bêtes sauvages occuperont le territoire, parmi lesquelles figurent encore différents types de quadrupèdes et d'oiseaux rapaces :

\section{'lhbqqbtn wtht lyšmn' 'hwh wthwy'rpd tl l[rbqy w] șby wšl w'rnb wšrn wṣdh w'r wqh w'l t'mr qr [yt']}

Que sa végétation soit arrachée en un désert! Et qu'Arpad devienne un monceau de ruines [pour servir de gîte ...] à la gazelle, et au renard ${ }^{13}$, et au lièvre, et au chat sauvage, et au hibou, et au gypaète et à la pie! Et qu'il ne soit plus fait mention de cette vil[le-là $]^{14}$.

12 Editio princeps Ronzevalle 1930-31 (Stèle I); Dupont-Sommer 196o; voir également Lemaire et Durand 1984, Fitzmyer 1995 b ; en dernier, Fales et Grassi 2016, p. 92-122.

$13 \breve{S}$ l, traduit comme «chacal » par Lemaire et Durand, est plus probablement à entendre comme «renard» car le terme est mieux attesté avec cette signification en sémitique. En outre, comme le suggère Giulia Grassi, il est fort possible que la distinction entre les deux espèces ne fût probablement pas pertinente en araméen ancien, étant donné que les autres mots araméens pour «chacal » sont plus tardifs. Voir, à ce sujet, Grassi 2019, p. 95-96; SED II, no. 237.

14 Sfiré I A, 32-33 (= KAI 222 A), trad. Lemaire et Durand 1984, légèrement modifiée. 
Les inscriptions royales d'époque assyrienne nous offrent des témoignages supplémentaires. Dans une lettre d'Assarhaddon adressée au dieu Aššur, le roi fait un rapport au dieu sur le territoire de Šubria, un ancien allié de l'empire assyrien qu'il vient de soumettre et dont les villes sont maintenant devenues un repaire «des renards et des hyènes $»^{15}$. On peut enfin citer l'inscription d'Assurbanipal qui célèbre sa victoire sur l'Elam. À la suite d'une liste où le roi indique les quantités de biens et d'animaux domestiques qu'il a fait déplacer de l'Elam vers l'Assyrie, il mentionne les bêtes qu'il va, en revanche, laisser sur le territoire d'Elam, lequel sera ainsi rempli « d'ânes sauvages, de gazelles et de bêtes des champs ${ }^{16}$.

Il semble donc que ce topos proche-oriental puisse être employé dans des situations différentes selon les exigences. On ne peut pas exclure que dans la Bible hébraïque le motif ait été appliqué originairement à Israël/Juda car la destruction qui frappe Jérusalem est perçue chez les prophètes comme une conséquence inévitable de la rupture de l'alliance entre le peuple et sa divinité tutélaire ${ }^{17}$. Toutefois, comme nous l'avons vu, les descriptions les plus détaillées ne concernent jamais Jérusalem, mais se trouvent toujours dans un contexte différent, à savoir celui des oracles contre les nations. Elles sont dirigées contre des villes étrangères, parmi lesquelles Babylone et Edom jouent un rôle de premier rang.

Bien qu'il soit impossible de reconstruire le contexte original de ces oracles ainsi que de proposer une datation absolue des passages concernés, l'on peut néanmoins établir quelques points de repère quant à la chronologie relative et à la présence de liens intertextuels explicites. La plupart des commentateurs est d'accord sur le fait que la destruction de Babylone annoncée en Jer 50,3940, puis évoquée en 51,37, reprend, d'une part, les oracles précédents du livre de Jérémie, et notamment l'oracle de Jer 49,17-21 contre Edom ${ }^{18}$, et, d'autre part, l'oracle d'Is 13 contre Babylone ${ }^{19}$. En outre, l'oracle d'Is 13 est retravaillé et élargi, notamment en ce qui concerne la liste des créatures des ruines en Is $34^{20}$. Ce dernier est considéré, à juste titre, comme l'un des textes les plus

\footnotetext{
15 Lettre à Aššur, K O2852 + K o9662, RINAP 4, Esarhaddon o33, ex. 2, i.e.1-6. Voir également Hillers 1964, p. 44.

16 PRISMA A vi 81-89; iv 96-106, RINAP 5, Asshurbanipal ol1.

17 Voir surtout en ce sens la fonction des malédictions en Deut 28 et ses parallèles proche-orientaux où n'apparaissent cependant pas les habitants des ruines. Sur les parallèles entre les malédictions de la Bible hébraïque et d'autres textes du Proche Orient ancien, voir par exemple Hempel 1925; Fensham 1963; Hillers 1964 ; García Recio 1995.

18 Voir par exemple Duhm 19o1, p. 366, McKane 1996, p. 1292-1293; Bellis 1999, p. 192-199.

19 Duhm 1901, p. 365-366; Lundbom 2004, p. 423.

20 Vermeylen 1977, p. 349-446; Gosse 199o, p. 399-402.
} 
tardifs dans la composition du recueil d'Isaïe, au point que les chapitres 34-35 (qui mettent en miroir le châtiment d'Edom et l'annonce de salut pour Sion) sont parfois connus comme la "petite apocalypse d'Isaïe», servant de charnière littéraire entre les trois parties du recueil ${ }^{21}$. Isaïe 13 et 34 se font ainsi clairement écho à l'intérieur du livre et le chapitre 34 est vraisemblablement le texte le plus récent de notre corpus, dont la composition pourrait se situer pendant l'époque perse ou au début de l'époque hellénistique ${ }^{22}$.

La relation de Sophonie 2 avec le reste des oracles est moins claire. Si le noyau du livre est d'habitude situé à la fin de la monarchie judéenne, donc vers la fin du VII ${ }^{\mathrm{e}}$ siècle, sa forme finale a fait l'objet d'un processus rédactionnel conséquent et plusieurs commentateurs estiment que l'oracle contre Ninive serait effectivement plus récent ${ }^{23}$. Il faut à cet égard observer que la série d'oiseaux $q \bar{a} a t+q i p p o \bar{d}$ mentionnée en Soph 2,14, et par ailleurs très rare, se retrouve à l'identique en Is 34,11. Au vu de la nature tardive et en partie composite d'Is 34, il me paraît plus probable que le rédacteur du passage isaïen ait, dans ce cas, emprunté à Sophonie, plutôt que l'inverse ${ }^{24}$. Les textes les plus anciens du corpus pourraient donc être représentés soit par l'oracle contre Babylone en Is 13, soit par celui contre Ninive en Soph 2. Toutefois, bien qu'il apparaisse clair que Is 34 et Jer 50 (et 51 ) reprennent des passages

21 La question de la rédaction des chapitres 34 et 35 est, en revanche, plus complexe. D'après certains commentateurs les deux chapitres ont la même origine, en ayant été conçus dès le début comme une unité (Watts 1985-1987, p. 2-18; Gosse 1990; Blenkinsopp 200o, p. 447-457); d'autres pensent, en revanche, qu'Is 34 constituait un texte apocalyptique à l'origine séparé (Kaiser 1980, p. 351-366); d'autres encore considèrent Is 35 comme le texte le plus tardif (voir en ce sens l'étude classique de Steck 1985; mais également Wildberger 1982, p. 1324-1351; pour une critique des arguments de Steck voir Mathews 1995). Beuken estime que les deux chapitres, à l'origine indépendants, ont progressivement été harmonisés l'un avec l'autre (Beuken 2000, p. 279-304; pour une vision similaire, voir aussi Childs 2001, p. 249-258). Sur la relation entre Is 13 et 34, voir également Gosse 1988, p. 159-169.

22 Pour une analyse plus détaillée voir Nihan 2017, p. 89-90, 108-111. Il est possible qu'Is 34,9-17 ait fait l'objet d'un travail rédactionnel supplémentaire, puisque l'indication de «chercher et lire dans le livre de Yhwh » au verset 16 paraît secondaire : voir à ce sujet Donner 1990.

23 Hagedorn 2011, p. 113-129. Contra Hubert Irsigler (2002, p. 217-218), pour lequel les versets $2,12^{*}, 13^{-14^{*}}$ remontent effectivement à l'époque josianique.

24 En Soph 2,13-15, le verset 15 pourrait être une glose ultérieure (Ben Zvi 1991, p. 183 ; Irsigler 2002, p. 298-301; Hagedorn 2011, p. 123, lequel pense toutefois que l'oracle d'Isaïe contre Edom et Babylone a été emprunté par le rédacteur de Sophonie et adressé secondairement à Ninive). Le texte de Soph 2,15, qui se réfère à la ville « qui demeurait en sécurité, qui disait dans son cœur, 'moi, et seulement moi!'» se retrouve à l'identique en Is 47,8 référant à Babylone, et le motif du sifflement est présent dans plusieurs passages de Jérémie. 
antérieurs de chaque recueil, les rapports entre Isaïe et Jérémie restent, eux, difficiles à comprendre ${ }^{25}$.

Face à la difficulté de reconstruire des directions d'intertextualité linéaires pour ces passages, deux remarques plus générales s'imposent. D'un côté, il faut garder à l'esprit que nous sommes en présence d'un topos du genre prophétique où des expressions similaires peuvent être appliquées à des territoires différents : par conséquent, il serait non seulement impossible, mais méthodologiquement douteux d'utiliser les liens intertextuels ou bien des éléments isolés de ces descriptions pour une datation des textes ${ }^{26}$. D'un autre côté, il paraît néanmoins possible d'affirmer qu'à un moment donné le topos des habitants des ruines a été associé de manière plus étroite - voire paradigmatique - à une ville spécifique, à savoir Babylone ${ }^{27}$. Il s'agit, en effet, de la cité la plus souvent nommée, à la fois en Jérémie et en Isaïe. Cette tradition est encore prolongée dans le livre de Baruch qui prédit la chute de la cité, désormais abandonnée et peuplée par des démons ${ }^{28}$ :

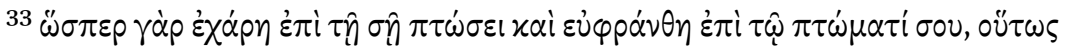

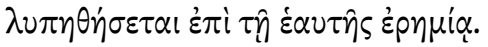

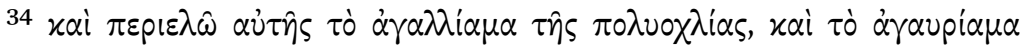

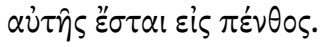

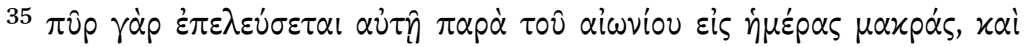

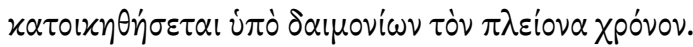

${ }^{33}$ Ainsi, tout comme elle (= Babylone) s'est réjouie de ta chute et s'est félicitée de ta ruine, de la même manière elle sera affligée de sa propre dévastation.

${ }^{34}$ Et je lui enlèverai la joie de la multitude de sa population, et son orgueil sera changé en deuil.

${ }^{35}$ Car un feu s'abattra sur elle de la part de l'Éternel pour de longs jours, et elle sera habitée pour plusieurs temps encore par des démons.

Alors que le feu descendant sur la ville évoque indirectement la chute de Sodome et Gomorrhe, les démons installés parmi les ruines de Babylone nous

\footnotetext{
25 Voir encore récemment à cet égard les conclusions de Beuken 2015.

26 Le problème renvoie, d'ailleurs, à la question plus large et qui ne peut pas être discutée ici, des relations entre les oracles aux nations parmi les différents recueils prophétiques ; sur ce sujet on peut voir, entre autres, Christensen 1989; Holt, Hyun Chul et Mein 2015.

27 Comme cela a été suggéré par Nihan 2017, p. 90.

28 Bar 4,33-35. Ce topos est également associé à Babylone dans le Nouveau Testament (Ap 18,2).
} 
rappellent ceux des malédictions sumériennes et paléo-babyloniennes. La permanence d'un tel motif dans un texte non antérieur au deuxième siècle av. n. è. ${ }^{29}$ est un indice de continuité considérable entres les traditions proche-orientales et les textes bibliques. Ces derniers montrent néanmoins plusieurs éléments d'innovation quant à l'usage de ce topos, qui méritent d'être observés plus en détail.

\subsection{Les isotopies de l'espace des ruines}

Dans l'étude de l'espace des ruines, les chercheurs se sont principalement concentrés, jusqu'à présent, sur des problèmes d'identification - certes importants - posés par les noms d'animaux mentionnés dans ces passages. Une autre question souvent soulevée concerne le «degré de démoniaque» qu'on peut attribuer à ces créatures : un tel questionnement a été suggéré, d'un côté, par la présence de Lilith parmi les habitants des ruines, et de l'autre côté, par l'introduction du mot $\delta \alpha \mu o ́ v i \alpha$ dans la LXX d'Isaïe ${ }^{30}$. En revanche, peu d'attention a été dédiée aux qualités propres à cet espace dont les habitants sont révélateurs. Ce dernier aspect a fait l'objet d'une analyse récente par Christophe Nihan ${ }^{31}$, à laquelle une grande partie des observations de cette section est redevable. Nihan a mis en évidence trois concepts clés qui me paraissent utiles pour comprendre la fonction des créatures qui peuplent l'espace des ruines, un espace qui n’appartient désormais plus à la culture, sans qu'il soit pour autant entièrement restitué à la nature, et qui est donc caractérisé par une forte marginalité et une dimension antisociale importante. Cette dimension est justement soulignée par les animaux qui occupent cet espace, qui ne sont pas les animaux « classiques » de l'espace sauvage auxquels on pourrait s'attendre, tels que, par exemple, lions, ours et panthères, ou tout simplement animaux des steppes (bien que ces derniers soient également présents). Il s'agit en revanche d'un mélange de créatures diverses, telles que boucs, canidés, plusieurs espèces de rapaces, ainsi que d'autres créatures parfois difficiles à identifier et pour lesquelles le dénominateur commun est à chercher ailleurs.

À cet égard, Nihan propose de lire ces passages prophétiques à la lumière des isotopies qu'ils dégagent. Or une isotopie est constituée par les éléments du discours permettant d'identifier dans un texte donné une série d'oppositions sémantiques qui renvoient à un contexte de référence commun. À la différence d'un champ lexical ou sémantique, les catégories sémantiques d'une isotopie ne sont pas définies par la signification primaire de chaque élément, mais seulement en vertu du contexte où ces éléments apparaissent. Cette

\footnotetext{
29 Pour la question de la Vorlage de Baruch, voir supra, p. 8o.

30 Voir, sur ce point, Janowski 1993; Blair 2009, p. 72-95; Frey-Anthes 2007, p. 159-241.

$31 \quad$ Nihan 2017.
} 
approche permet donc de dépasser les difficultés, parfois insurmontables, liées à l'identification des espèces animales et d'autres termes problématiques, et d'étudier leur fonctionnement comme un ensemble, en se concentrant surtout sur la caractérisation de l'espace qu'ils occupent. Cet espace est en relation étroite avec la souillure et l'impureté car une bonne partie des oiseaux des ruines se retrouvent dans les listes d'animaux impurs du Lévitique (chapitre 11) et du Deutéronome (chapitre 14). Il est, en outre, associé à la mort et au chaos, auquel il est explicitement fait référence en Is 34,11 («YHWH étendra sur elle le cordeau du vide et la pierre à mesurer le chaos »), et qui est également évoqué par l'image du désert (par exemple en Soph 2,13: «Yhwh ... fera de Ninive une dévastation, aride comme le désert») ainsi que par l'expression «repaire de tannim $»^{32}$. La dimension sonore qui envahit les ruines est, en outre, mise en avant à travers l'insistance sur les cris puissants produits par ses habitants (Soph 2,14: «Le bruit se fait entendre à la fenêtre »; Is 13,22: «les 'hurleurs' crieront dans ses palais et les chacals dans ses résidences de luxe »; Is 34,14: «Les créatures du désert et les hurleurs s'y rencontreront, chaque boucy appellera son compagnon »). Cette dernière donnée est d'autant plus intéressante que, comme nous l'avons vu, elle est déjà présente dans les descriptions de ruines d'autres textes proche-orientaux, mais également parce que dans tout le Proche Orient ancien les cris et les hurlements sont des qualités typiquement attribuées aux démons. C'est notamment pour souligner cet aspect sonore que j'adopte ici la traduction proposée par Nihan de 'iyyîm par « hurleurs ». Ce terme, attesté seulement trois fois dans la Bible hébraïque, et toujours dans le contexte des ruines ${ }^{33}$, indique probablement un canidé sauvage, hyène, chacal ou renard, et possède un certain nombre de parallèles dans d'autres langues chamito-sémitiques (notamment syriaque, arabe, égyptien) ${ }^{34}$. Une origine onomatopéique est parfois proposée. Alors que celle-ci n'aide pas nécessairement à l'identification de l'espèce en hébreu, il est néanmoins envisageable qu'il y ait eu un lien chez les anciens parlers entre le nom de l'animal et son cri, d'autant plus qu'en hébreu ' $y$ apparait également comme forme courte de l'interjection 'ôy («hélas!»).

Deuxièmement, Nihan souligne la nature hétérogène et potentiellement ouverte de l'ensemble formé par les habitants des ruines. Un tel ensemble comprend non seulement des animaux, mais également des puissances, dont certaines sont facilement identifiables en vertu de leur arrière-plan

32 Voir, en ce sens, Ps 44,20: « tu nous a écrasés dans un endroit de tannîm et recouvert avec l'ombre de la mort », et Nihan 2017, p. 100.

33 Is 13,$22 ; 34,14 ;$ Jer 50,39.

34 Voir SED II, no. 21. D'après les lexicographes arabes, l'animal désigné comme 'íbnu 'āwa est considéré « comme un mélange né d'un chien et d'un renard». À remarquer également l'égyptien $j w / j w j w=$ «type de chien » (Erman-Grapow 1926, I, 48, 50). 
proche-oriental, comme la démone Lilith en Is $34,14^{35}$, alors que d'autres demeurent plus obscures. Le caractère mixte de cet ensemble est aussi signalé par la présence des șiyyîm en Isaïe $(13,21 ; 34,14$, mais voir également Is 23,13$)$ et en Jérémie $\left(5^{\circ}, 39\right)$, un terme que j'ai, ici, choisi de traduire par «créatures du désert». Les dictionnaires considèrent le substantif comme ambigu ${ }^{36}$ et les parallèles sémitiques pour la signification de «chat sauvage » qui lui est parfois attribuée sont maigres ${ }^{37}$. En dehors du contexte des ruines, les occurrences de ce terme, toujours au pluriel, ne semblent pas pouvoir se référer à une espèce zoologique précise. Il semble plutôt indiquer un groupe social résidant dans les steppes, notamment en Ps $72,9^{38}$, ou un ensemble de puissances du désert. Il apparaît, en outre, dans un contexte mythologique clair en tant que groupe auquel Yhwh donne à manger les restes du Léviathan qu'il vient de tuer (Ps 74,14) ${ }^{39}$.

Si l'on ajoute à ces données le fait que les démons prennent volontiers l'aspect d'oiseaux dans les traditions levantines et proche-orientales, on ne s'étonnera pas de voir démons et oiseaux mentionnés ensemble ici. On peut donc en conclure que la distinction entre démons et animaux dans l'espace des ruines n'est pas si nette et que, au contraire, l'hétérogénéité de ce paysage est un élément essentiel de sa caractérisation en tant qu'espace marginal et dystopique.

Finalement, Nihan souligne le caractère exceptionnel d'Is 34 parmi les descriptions de ruines. Contrairement aux autres passages bibliques et au reste des traditions proche-orientales où les dieux abandonnent à jamais les terres vouées à la ruine, dans l'oracle contre Edom Yhwh continue à exercer une forme de souveraineté sur ce territoire, comme le souligne le verset 17 ( «c'est la bouche de Yhwh qui l'ordonne, c'est son esprit qui les rassemble, c'est lui qui a procédé au tirage des parts pour eux, et c'est sa main qui leur a partagé le pays au cordeau »). C'est ainsi que cet espace chaotique et étranger à l'homme peut redevenir un lieu de repos et de tranquillité pour ses nouveaux habitants ( « c'est pour toujours qu'elles en prendront possession, de génération en génération elles y résideront »). Cette dernière remarque a des implications importantes pour comprendre la lecture d'Is 34 par le traducteur grec. Toutefois, avant d'analyser l'exégèse grecque de ce chapitre, il faut explorer la question de l'identification des habitants des ruines par les traducteurs.

35 Pour une discussion sur l'arrière-plan proche-oriental de Lilith, voir §3.2.4.

36 Gesenius 1916, s.v. șî; HALOT, s.v. șî II . Voir en outre H.-P. Müller 2003.

37 Comme le relèvent d'ailleurs Militarev et Kogan (SED II, no. 224), qui citent l'Arabe daywan et considèrent la forme protosémitique comme «hautement incertaine ».

38 Le Ps 72,9 mentionne les șiyyîm qui se prosternent devant Yhwh, à côté des rois des nations et des chefs de pays exotiques, comme Tarsis (vv. 10-11).

39 Nihan 2017, p. 106-107. Sur ces passages, voir également Janowski 1993, p. 161-162; Frey-Anthes 2007, p. 173-175. 
2 Des oiseaux, des boucs et des serpents : sur quelques problèmes de correspondance entre hébreu et grec

Comme nous l'avons vu précédemment, le paysage des ruines comprend plusieurs espèces animales : reptiles, rapaces, canidés du désert, boucs sauvages. Alors que certaines sont bien connues et bien attestées (comme, par exemple, le bouc, śấîr, ou le corbeau, 'ôrēb), d'autres posent de nombreux problèmes d'identification. Il s'agit d'un vocabulaire spécialisé et composé pour la plupart de mots rares. L'analyse des équivalents grecs démontre que les traducteurs éprouvaient déjà quelques difficultés à son encontre. On essayera donc de se poser la question de la signification de ces noms d'animaux du point de vue des traducteurs anciens. Un bon exemple des difficultés soulevées par ce lexique est représenté par l'oracle contre l'Assyrie dans la LXX de Soph 2,13-14:

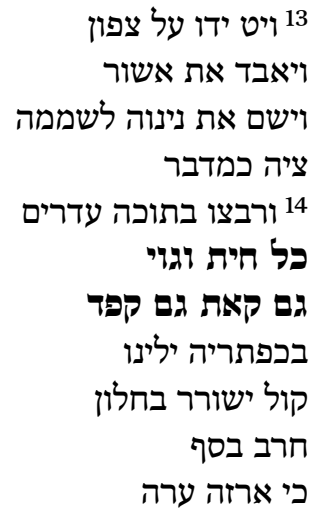

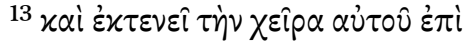

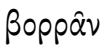

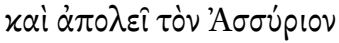

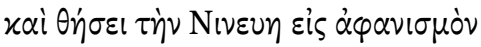

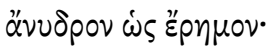

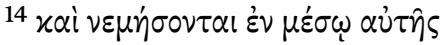

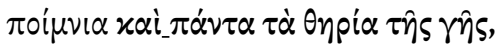

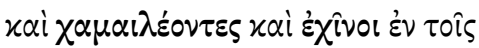

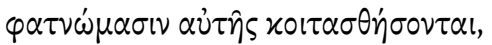

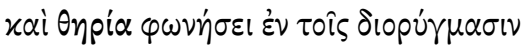

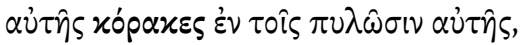

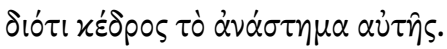

${ }^{13}$ Et il étendra la main contre le Nord, détruira l'Assyrien et réduira à l'anéantissement Ninive, privée d'eau comme un désert.

14 Des troupeaux iront paître au milieu d'elle

et toutes les bêtes sauvages de la terre, et caméléons et hérissons passeront la nuit dans les caissons, et des bêtes sauvages crieront dans ses canaux, des corbeaux sur ses portes, car sa structure est en cèdre. 
Alors que la vision d'ensemble reste similaire en hébreu et en grec, nous montrant un territoire occupé par les bêtes sauvages et l'intérieur d'une maison luxueuse remplie d'animaux jetant des cris lugubres, nombre de détails divergent. Parmi les plus évidents, on peut mentionner la répétition de $\theta \eta p i \alpha$ au verset $14 \mathrm{~b} \alpha$, qui n'a pas d'équivalent en hébreu ni dans les versions anciennes et qui est peut-être due à un souci de clarification du passage, ainsi que la mention

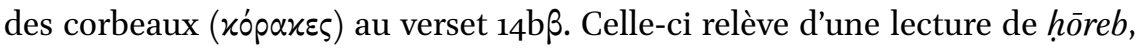
« ruine » ou « décombres », comme 'ôrēb. Ce choix de traduction est également adopté par Jérôme qui nous informe sur la diversité des versions attestées pour ce passage encore à son époque ${ }^{40}$. Hrb est lectio difficilior, et la leçon est attestée par toute la tradition massorétique : elle a l'appui du rouleau des Douze retrouvé dans les grottes de Murabbaat, proche du texte massorétique ${ }^{41}$ ainsi que d'une version targumique ${ }^{42}$. Aquila et Symmaque lisent hereb, «épée »,

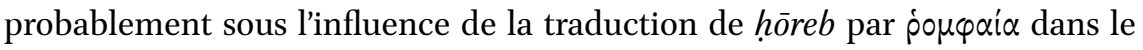
texte grec d'Ag 1,11. Il est, en revanche, probable que la Septante conserve la leçon originale ('rb) car la mention du corbeau est bien encadrée à la fois par la référence aux cris à la fenêtre, qui précède, et aux portes arrachées permettant l'entrée des oiseaux, qui suit ${ }^{43}$. Les oiseaux, qui occupent ainsi tout le périmètre de la maison, sont en effet dominants dans ce type de paysage, et il est probable que qāat autant que qippôd en Soph 2,14 fassent référence à des rapaces. Qäat figure sur la liste des oiseaux impurs de Lev 11 et Deut 14 ainsi que dans le Ps 102,7 et en Is 34,11. Même si l'identification proposée par Driver du $q \bar{a}$ 'at avec un rapace de la famille des strigidae (l'otus, ou petit-duc) n'est pas assurée ${ }^{44}$, sa position dans la liste des oiseaux impurs du Pentateuque suggère qu'il s'agit effectivement d'un petit rapace. Cela nous amène à un problème supplémentaire rencontré par le traducteur de Sophonie. Comme le montre le tableau suivant (tableau 1), qāat a été généralement compris comme oiseau dans ses autres occurrences, même si les identifications divergent:

40 Jer., Comm. in duodecim prophetes, ad loc.

41 Mur, col. xx, l. 29 (Benoit et al. 1961, DJD 2, p. 200-201).

42 Targum Jonathan en Soph 2,14.

43 Voir Nihan 2017, p. 94, note 16, avec Irsigler (2002) et autres. Le passage de 'rb vers hrb pourrait facilement être expliqué par une faute de transmission, produite par la proximité sonore entre les deux lexèmes. Contra Barthélemy 1992, p. 897-89, qui donne à hōreb le sens de «décombres ». Cette signification n'est toutefois jamais attestée ailleurs pour hōreb, à l'exception d'Is 61,4, où le mot se trouve au pluriel. En revanche, on ne comprend pas exactement ce qu'une décombre pourrait signifier. Je remercie vivement Jan Rückl pour son aide pour la compréhension de ce passage.

44 Driver 1955 I, p. 16. Pour une critique de la méthode d'identification des oiseaux adoptée par Driver, fondée en grande partie sur l'onomatopée et l'étymologie des racines, voir Kogan 2015 ; Angelini et Nihan 2020. 
TABLEAU 1 Équivalents grecs de $q \bar{a} a t$

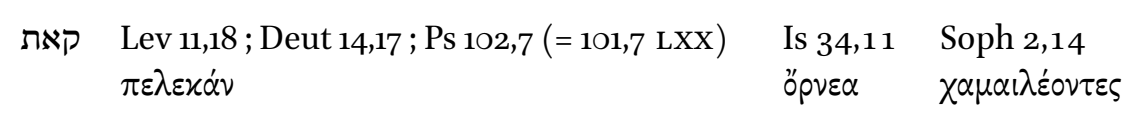

La traduction par $\pi \varepsilon \lambda \varepsilon x \dot{\alpha} \nu$ attestée dans le Pentateuque ${ }^{45}$ dérive peut-être d'une lecture étymologique de la racine $q^{\prime} t$, «vomir», en référence à la croyance, déjà répandue dans l'Antiquitée ${ }^{46}$, selon laquelle le pélican rejette la nourriture de sa bouche pour nourrir ses petits. La version du Pentateuque a été suivie par le traducteur des Psaumes, alors que le traducteur d'Isaïe a choisi le mot plus générique d'« oiseaux ». Ces choix démontrent que les traducteurs grecs avaient reconnu en q't un oiseau, même s'ils n'étaient plus en mesure d'en identifier l'espèce. La traduction par « caméléon » en Sophonie est en revanche le produit d'un choix différent. Elle pourrait être motivée par un rapprochement phonétique entre $q$ 't et $k h$, ce dernier étant le nom d'un petit reptile impur présent en Lev 11,30 et traduit par $\chi \alpha \mu \alpha i \lambda \varepsilon \dot{\varepsilon} \omega \nu^{47}$. En outre, le camé-

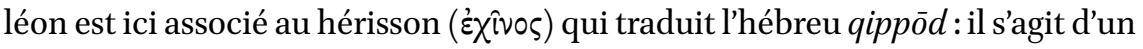
animal typique des ruines d'après Is 14,23 («je ferai de Babylone une possession du qippōd») et il apparaît également en Is 34,11. Il est toujours compris

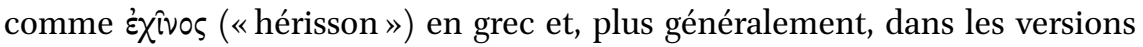
anciennes. Le traducteur a probablement interprété le mot sur la base de la racine hébraïque $q p d$, «être bouclé » ou « s'enrouler » et cette signification est parfois déjà assignée au zoonyme hébreu ${ }^{48}$. Elle pose, néanmoins, problème dans le cas de Sophonie 2 où le contexte semble fortement indiquer qu'il s'agit d'un oiseau : on comprend sinon mal comment des hérissons pourraient passer

45 La plupart des manuscrits de Deut 14,17 ont $x \alpha \tau \alpha p \rho \alpha \dot{x} \tau \eta \varsigma$ en correspondance de $q^{\prime} t$. Nihan et moi-même avons, toutefois, démontré ailleurs que la liste des oiseaux en hébreu suivait, à l'origine, un ordre différent en Lévitique et Deutéronome, et que même dans les versions grecques du Deutéronome $\pi \varepsilon \lambda \varepsilon \alpha_{\alpha} \nu$ devait traduire $q^{\prime} t$ : voir Angelini et Nihan 2020, p. 56-57.

46 Par exemple Ael., NA 3, 20 et 23.

47 Le rapprochement phonétique apparaît d'autant plus vraisemblable si l'on considère que $q^{\prime} t$ pouvait apparaître aux traducteurs comme l'état construit d'une forme originelle $q^{\prime} h$.

48 Par exemple Gesenius 1916, s.v. qippōd; Zurro Rodriguez 1995, p. 534-535. En sémitique les parallèles sont attestés à la fois pour «hérisson » (notamment l'éblaïte qippadum) et pour « rapace» (le syriaque qwpda', voir Payne Smith 1879, p. 3687-3688). Comme l'expliquent Alexander Militarev et Leonid Kogan (SED II, no. 133) à partir du sémitique qpd on attendrait en hébreu une fricative $(z / \underline{d})$. Or, la forme qippôz est effectivement attestée comme hapax en Is 34,15 , mais là encore le contexte, où il est question de la ponte des œufs, empêche l'identification avec le hérisson. Voir infra, p. 166, note 62. 
la nuit en haut des chapiteaux. À cet égard, il convient de remarquer que le problème a été résolu en grec par l'introduction des $\varphi \alpha \tau \nu \omega ́ \mu \alpha \sigma i v$, «caisses » ou «caissons $»^{49}$ qui traduit exceptionnellement l'hébreu kaptōr: ainsi, au lieu des oiseaux nichant aux sommets des colonnes, on a en grec l'image de petites bêtes rampant dans les caisses.

Les difficultés soulevées par l'identification des animaux dans ce passage pourraient facilement être élargies au reste de la faune comprise sous la rubrique «animaux des ruines ». Ce lexique mériterait une analyse systématique qui nous amènerait cependant trop loin du cadre de l'enquête sur les démons ${ }^{50}$. Ainsi, je ne discuterai que de quelques cas directement pertinents à la question des démons. La Septante d'Isaïe s'avère, à cet égard, un texte clé, notamment par son caractère interprétatif considérable, ainsi que par une attention particulière prêtée par le traducteur à la langue cible ${ }^{51}$.

\subsection{Les habitants des ruines et le bestiaire d'Isaïe}

En observant la traduction des habitants des ruines dans le livre d'Isaïe, on reste frappé par plusieurs équivalences posées par le traducteur. Celle qui nous servira d'entrée pour l'analyse du dossier est la traduction de śa îrîm, «boucs

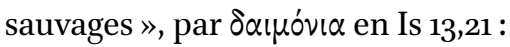

ורבצו שם יעיים

ומלאו בתיהם אחים

ושכנו שם בנות יענה

ושעיירים ירקדו שם בעות יענות

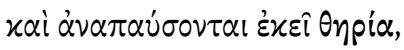

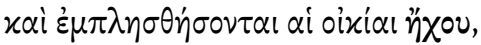

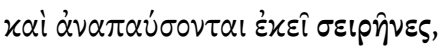

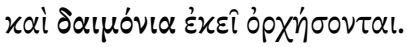

Et se reposeront là-bas des bêtes sauvages les maisons seront remplies de bruit, se reposeront là-bas des sirènes, et des démons danseront là-bas.

49 Dogniez et al. 1999, p. 356.

50 Voir jusqu'à présent Nihan 2017 ; Angelini et Nihan, 2020.

51 La littérature sur la nature «libre » de la traduction du livre d'Isaïe ainsi que sur ses tendances actualisantes est désormais vaste. Parmi les études fondamentales on peut voir : Ziegler 1934; Seeligmann 1948 (republié dans Hanhart et Spieckermann 2004, p. 119-294); Schweitzer 2004; Troxel 2008; Dogniez 2010; Lee 2014. Sur l'Erfüllungsinterpretation, le procédé exégétique selon lequel le traducteur grec a interprété des prophéties du livre d'Isaïe comme se réalisant à son époque, voir, entre autres, Seeligmann 1948, p. 94-121 ; Hanhart 1983 a (republié dans Hanhart et Kratz 1999, p. 95-109) ; l'étude, désormais classique, de Van der Kooij 1998 (surtout p. 88-109) et Wagner 2013. Sur les écarts quantitatifs entre hébreu et grec voir désormais Van der Vorm-Croughs 2014. 
Ce passage est particulièrement intéressant, non seulement, car le traducteur introduit ici pour la première fois des démons dans le texte grec, mais, également, car le verset nous renvoie à la question, souvent discutée, de la nature démoniaque des śa îrîm dans le texte hébreu. Les śa îrîm apparaissent deux fois dans la Bible hébraïque, toujours au pluriel, comme objet d'un culte interdit. D'abord ils sont nommés en Lev 17,7 où la loi de centralisation du sacrifice sanglant vient interrompre le sacrifice que les Israélites avaient l'habitude d'offrir aux śâîîm, auxquels était rendu un culte « de prostitués » (zōnîm 'ahărêhem) dans les champs ouverts. Ils sont ensuite mentionnés en 2 Ch 11,15 avec les autels et les images en forme de veau que le roi Jéroboam se fabrique et auxquels il rend un culte illégitime :

\section{ויעמד לו כהנים לבמות ולשעירים ולעגלים אשר עשה}

Et il institua ses prêtres pour les hauts lieux, les boucs et les veaux qu'il avait fabriqués.

À cet égard, il faut souligner que le rapprochement entre les śốrîm et les satyres de la tradition grecque, qu'on a souvent justifié par l'élément capridé qu'ils partagent, est mal fondé. Tout d'abord, les satyres en Grèce ne font quasiment jamais l'objet d'un culte, au contraire des śâîîm; puis, comme l'ont justement rappelé Bernd Janowski et plus récemment Henrike Frey-Anthes ${ }^{52}$, nous ne sommes pas en mesure de nous faire une représentation quelconque d'un possible aspect hybride qui serait propre aux śa îrîm et qui les rapprocherait, donc, des satyres. Les plaquettes en ivoire de Megiddo, souvent utilisées en ce sens car elles montrent des hybrides ailés à tête humaine qui interagissent avec des capridés, ne doivent pas être interprétées à la lumière des catégories grecques ${ }^{53}$. Elles renvoient par contre au motif mésopotamien de la lutte contre les créatures appélées Mischwesen. Ces objets de luxe - et non de culte - démontrent donc, d'une part, que ce sujet décoratif était connu en Palestine et, d'autre part, que l'élément caprin est une composante récurrente

$5^{2} \quad$ Voir Janowski et Wilhelm 1993, p. 120-123; Janowski 1999 b, p. 732-733; Frey-Anthes 2007, p. 203-208 (et p. 313, image no. 45). L'image a parfois été associée également à Azazel, tout d'abord par Otto Eissfeld, et ensuite par Othmar Keel et Silvia Schroer (voir par exemple Keel 1997, p. 83-84, fig. 96-97 et bibliographie relative). L'idée qu'Azazel ait eu lui-même une apparence hybride et/ou caprine a été également proposée par Levine 1989, p. 251 et Milgrom 1991, p. 1021.

Loud 1939, plaque 5 , no. 4-5. 
des Mischwesen car il est lié à la dimension de la force sauvage non maitrisée bien exprimée par l'image des capridés au Levant ${ }^{54}$.

Il faut, en outre, éviter de lire trop rapidement les śoîrîm du livre d'Isaïe à la lumière de Lev 17,7 et de $2 \mathrm{Ch}$ 11,15 et chercher davantage à interpréter chaque passage dans son contexte. En ce qui concerne le chapitre 17 du Lévitique, Julia Rhyder a récemment montré que les śaîrî̀m destinataires des sacrifices dans les champs répondent ici à la dynamique spatiale qui traverse tout le chapitre et qui oppose centre et périphérie : ils servent notamment à marquer un espace marginal. Elle a, en outre, suggéré que les śaîrîm de Lev 17 sont à mettre en relation avec la mention d'Azazel au chapitre 16, lui aussi destinataire d'un bouc (śá $\hat{i} r)$ lors du rite d'élimination ${ }^{55}$. Comme Azazel, les śs ${ }^{0} \hat{r} r i m$ sont les « êtres périphériques » par excellence, associés à un espace chaotique, décentralisé et donc impur du point de vue cultuel ${ }^{56}$. Bien qu'ayant des connotations démoniaques, étant donnée leur proximité avec Azazel avec lequel ils partagent précisément l'espace, ils ne correspondent pas nécessairement à une réalité cultuelle de l'époque, mais feraient plutôt partie de la stratégie du rédacteur pour lier entre eux les chapitres 16 et 17 , qui relèvent à l'origine de deux couches littéraires différentes ${ }^{57}$. Pour sa part, le rédacteur du passage des Chroniques réinterprète les boucs du Lévitique en les associant à toute une série d'objets et d'images divines qui rentrent désormais sous la catégorie d'idoles, comme les «haut lieux » et les « veaux ». De ce point de vue, il est en accord avec les traditions attestées dans la LXX, qui traduisent śaîirim à la fois en Lev 17,7 et en 2 Ch 11,15 par $\mu \alpha \dot{\tau} \tau \alpha$ lol (« objets vains »), un terme important du lexique grec dans la polémique contre les idoles ${ }^{58}$.

Par contre, dans le cas d'Is 13,21 le contexte n'a rien à voir avec des idoles, ni avec des pratiques cultuelles quelconques et suggère une interprétation des śaîrim tout simplement comme des «boucs sauvages», animaux qui s'inscrivent très bien à l'intérieur du paysage des ruines. Néanmoins un aspect démoniaque, ou plutôt, comme le dit Nihan, un «potentiel démoniaque » 59 , est présent dans ce bouc : comme je l'ai mis en évidence plus haut, il est lié à la nature chaotique et antisociale de l'espace des ruines lui-même.

\footnotetext{
54 Voir Keel et Uehlinger 2001 § 10-12, p. 29-33; Frey-Anthes 2007, p. 203-208.

55 Voir supra, § 3.2.1.

$5^{6}$ Rhyder 2019, p. 205-214.

57 Ibid., p. 240-249.

58 Voir infra, § 6.3. Le traducteur des Chroniques prolonge ce lien entre boucs et idoles

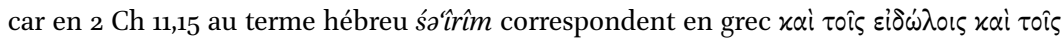

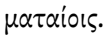

Nihan 2017, p. 107.
} 
Ce potentiel a bien été compris par le traducteur grec qui l'a rendu explicite en remplaçant des boucs bondissants par des démons qui dansent ${ }^{60}$. Pourtant son choix n'est pas motivé par une identification spécifique des boucs sauvages à des démons, mais par l'interprétation du paysage des ruines dans son ensemble. Pour comprendre la manière de procéder du traducteur, il faut alors élargir l'analyse à toutes les équivalences entre hébreu et grec et porter un regard comparatif sur les listes d'Is 13,21-22 et 34, notamment sur les versets 11, 13 et 14. Le tableau suivant (tableau 2) donne une vue d'ensemble de ces équivalences :

TABLEAU 2 Correspondances hébreu-grec (Is 13 et 34 ; Jer 50)

\begin{tabular}{|c|c|c|}
\hline 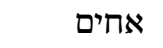 & $\hat{\eta} \times \circ \varsigma_{5}$ & Is 13,21 (hapax) \\
\hline \multirow[t]{3}{*}{ ציים } & Onpia & Is 13,21 \\
\hline & $\delta \alpha l \mu o ́ v i \alpha ?$ & Is 34,14 \\
\hline & $i \nu \delta \alpha \lambda \lambda \mu \alpha \tau \alpha$ & Jer $5^{\circ, 39}$ \\
\hline \multirow[t]{2}{*}{ תנים } & ĖXî้ol & Is 13,22 \\
\hline & 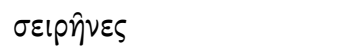 & Is 34,13 \\
\hline \multirow[t]{2}{*}{ בנות יענה } & 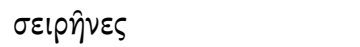 & Is 13,21 \\
\hline & $\sigma \tau p u \theta o i ́$ & Is 34,13 \\
\hline \multirow[t]{2}{*}{ שעירים } & $\delta \alpha ı o^{\prime} \downarrow l \alpha$ & Is 13,21 \\
\hline & 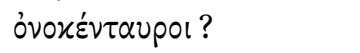 & Is 34,14 \\
\hline \multirow[t]{2}{*}{ איים } & 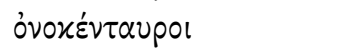 & Is 13,22 \\
\hline & 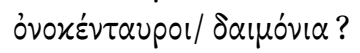 & Is 34,14 \\
\hline 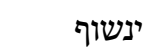 & 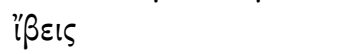 & Is 34,11 \\
\hline לילית & 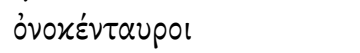 & Is 34,14 (hapax) \\
\hline קפוז & ExXivos & Is 34,15 (hapax) \\
\hline 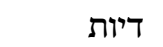 & $\varepsilon ُ \lambda \alpha \varphi \circ l$ & Is 34,15 \\
\hline
\end{tabular}

Tout d'abord, nous observons que des tendances variées et non nécessairement cohérentes entre elles sont à l'œuvre. Elles répondent aux différents problèmes posés par le texte hébreu. Nous repérons, ainsi, des choix qui visent à garder une certaine fidélité formelle. C'est le cas de l'hapax hébreu 'ôhîm qui

6o Le verbe rqd, à la fois «bondir» et «danser», en Is 13,21 fait référence aux bonds des boucs et décrit tout simplement le mouvement de l'animal : cette racine est appliquée aux jeunes veaux et aux béliers en Ps 29,$6 ; 114,4$ et 6 . L'équivalence avec ỏpxéopal, qui fait davantage référence à la danse, ne se trouve en revanche qu'ici et dans la LXX de 1 Ch 15,29 (pour la danse de David devant l'arche, comparer aussi avec le texte grec de $2 \mathrm{~S} 6,21$ ). 
apparaît dans la liste d'Is 13,21 (TM : « et les 'ōhîm envahiront leur maison ») et qui pourrait indiquer soit un oiseau rapace, soit une espèce de hyène ${ }^{61}$. N'étant vraisemblablement plus compris, il est transcrit par le grec $\hat{\eta} \times 05$, «écho » ou, dans ce cas, «bruit» (LXX : « et les maisons seront remplies de bruit»). Cette transcription rusée qui aboutit à un mot grec doué de signification propre rend explicite la dimension sonore du passage, qui était également importante dans l'original, d'autant plus que le verset suivant mentionne les hurlements lancés par les 'ayyîm et les tannîm dans les palais abandonnés de Babylone.

Dans d'autres cas, cependant, ce sont des règles de traduction différentes qui s'appliquent. Ainsi, par exemple, lorsque les oiseaux qui figurent parmi les habitants des ruines sont mentionnés dans les listes d'oiseaux impurs du Lévitique ou du Deutéronome, c'est alors de préférence la traduction grecque du Pentateuque qui fait autorité : c'est le cas de yanšôp en Is 34,11a, possiblement « effraie », qui est traduit par « ibis » sur la base de la Septante de Lev 11,17 et de Deut 14,16:

TM : «La chouette (qâa'at) et l'outarde (qippôd) y habiteront, l'effraie (yanšôp) et le corbeau y résideront».

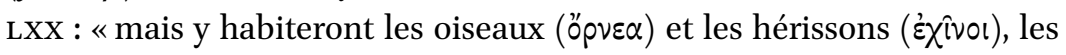
ibis ("ß $\beta \varepsilon ı \varsigma)$ et les corbeaux ».

Il ne s'agit toutefois pas d'une règle stricte. Nous avons déjà remarqué que, dans ce même verset, le terme q't est rendu par un générique « oiseaux », alors qu'il est traduit par «pélican » dans le Pentateuque. J'ai également déjà discuté la lecture étymologique de la racine qpd qui explique la traduction par éxivos. Le même choix a été fait par le traducteur de Sophonie et cette interprétation se retrouve dans les Targums ainsi que dans d'autres traductions anciennes: elle devait, donc, probablement correspondre à une exégèse courante de l'époque.

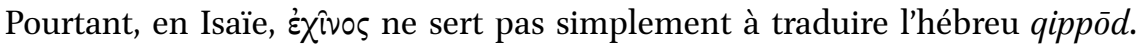
On le retrouve en Is 34,15 comme équivalent de l'hapax qippôz qui pourrait désigner soit un rapace, soit un type de reptile ${ }^{62}$, et qui a probablement été

61 Une espèce de hibou d'après Gesenius 1916, s.v. 'ōah ; une hyène d'après Driver 1955 II, p. 134 note 9, qui conteste le rapprochement souvent proposé entre l'hébreu *ōaḥ et l'accadien aḥ̂. Edwin Firmage (1991) considère également que le substantif signifie « hyène ». Pour une discussion du substantif, voir Riede 2002, p. 198-199.

62 Serpens iaculus d'après Gesenius (1916, s.v. qippôz), qui interprète le zoonyme par une lecture étymologique de la racine ( $q p z$, « se comprimer», en référence au mouvement de l'animal qui bondit de la terre sur les arbres); ainsi нАLOT, s.v., propose la significa-

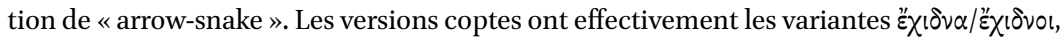

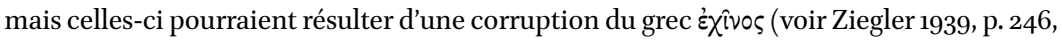


assimilé au qippōd au vu de la similarité phonétique entre les deux mots. En outre, é⿱亠乂 ivo apparaît encore sans motivation évidente en Is 13,22a pour rendre l'hébreu tannîm. Si l'identification des tannîm, comme je l'ai relevé plus haut, n'est pas complètement assurée, l'on peut par contre certainement exclure qu'il indique un hérisson. Ce terme n'est jamais traduit de manière systématique ni dans la LXX ni dans les versions anciennes, ce qui témoigne du fait que sa signification était désormais perdue à l'époque hellénistique et qu'elle était reconstruite à chaque fois, sur une base purement contextuelle ${ }^{63}$ :

TM : « et les chacals (tannîm) (crieront) dans ses résidences de luxe». LXX : «les hérissons ( $\dot{\chi} \chi i v o \iota)$ feront leurs nids dans leurs demeures».

L'ensemble de ces correspondances montre que, à l'exception de quelques cas de translittération ou de choix fondés sur le lexique du Pentateuque (notamment les listes d'animaux impurs du Lévitique), le traducteur tend à employer à peu près la même série de créatures dans tous les passages concernés, mais non de manière cohérente ou systématique. Nous pouvons détecter encore d'autres exemples : outre l'alternance entre sirènes et autruches, qui nous occupera dans le paragraphe suivant, le terme grec ỏvox́์vtavpos, faisant référence à un hybride («âne-centaure »), est employé pour rendre les 'ìyîm en Is 13,22a :

TM : «les hurleurs ('iyyîm) crieront dans leurs palais ».

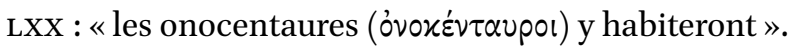

Mais il sert également en Is 34,14b pour traduire Lilith :

$\mathrm{TM}:$ « c'est encore là que résidera Lilith $(l \hat{l} \hat{l} \hat{i})$

et qu'elle se trouvera un lieu de repos».

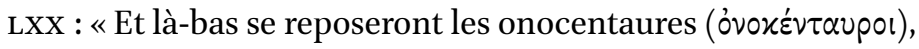
car ils trouveront leur repos ».

L'expression « les onocentaures habiteront là-bas » apparaît en outre en Is 34,11, là où l'hébreu donne 'bny $b h w$ « les pierres à mesurer le chaos ». Ziegler y voit une glose ${ }^{64}$ alors que d'autres reconstructions envisagent la traduction libre

apparatus I). Le rouleau d'Isaïe à Qumrân a une graphie différente (qwpd: 4QIsa ${ }^{\mathrm{a} X V I I I}$ 15). D'après Driver 1955 II, p. 136 il s'agit par contre d'un oiseau. Pour une discussion, voir, en outre, SED II, no. 133 .

63 Comparer, par exemple, encore avec Is 35,7 où tannîm est (peut-être) traduit par « oiseaux ».

64 Ziegler 1934, p. 7o. 
d'une expression originaire bny bhw «les fils du chaos » qui aurait ensuite été tardivement corrigée dans le $\mathrm{TM}^{65}$.

Même le terme $\delta \alpha \mu o ́ v i \alpha$ n'est pas réservé exclusivement aux śa'îrîm. Il réapparaît en Is 34,14 avec une correspondance peu claire puisqu'à trois termes hébreux ne correspondent que deux noms en grec:

TM : « Les créatures du désert (șiyyîm) et les hurleurs ('iyyîm) s'y rencontreront, chaque bouc (śáîr) appellera son compagnon».

LXX : «Et des démons ( $\delta \alpha \mu o ́ v i \alpha)$ se rencontreront avec des onocentaures

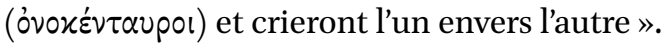

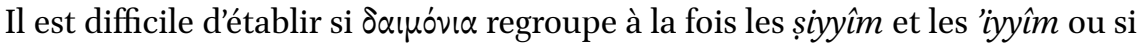

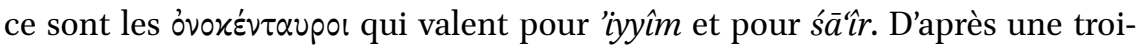

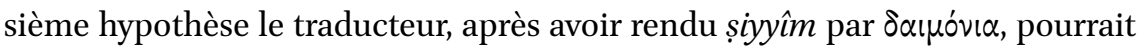
avoir estimé que śâîr rentrait dans la même catégorie, étant donné qu'il avait déjà traduit śấîr par $\delta \alpha \iota o^{\prime} v \alpha$ en Is 13,21, et que, par conséquent, il n'était pas nécessaire de répéter le mot deux fois ${ }^{66}$. Siyyîm aussi n’a pas de traduction systématique dans la LXx : il est rendu par Өńpı $\alpha$, «bêtes farouches » en Is 13,21, et par i $\nu \delta \alpha \dot{\lambda} \mu \alpha \tau \alpha$, «spectres », «images sans consistance», dans le texte grec de Jérémie, qui a un verset presque identique ${ }^{67}$ :

TM : « C'est pourquoi les créatures du désert (șiyyîm) y habiteront avec les hurleurs ('iyyîm)».

LXX : «C'est pourquoi les spectres (ivód $\lambda \mu \alpha \tau \alpha)$ habiteront dans les îles ».

La comparaison avec le texte grec de Jérémie s'avère d'autant plus intéressante que le style du traducteur diffère considérablement de celui d'Isaïe car il adopte une attitude beaucoup plus littérale. Par exemple, ne reconnaissant probablement pas le référent zoologique derrière 'iyyîm, il le traduit comme si c'était son homophone ' $\hat{\imath}$, «île». Néanmoins, le traducteur souligne également l'aspect démoniaque du paysage à travers la mention des ivód $\lambda \mu \alpha \tau \alpha$, «apparitions », «spectres », un choix qui est certainement en accord avec la traduction proposée par Isaïe ${ }^{68}$.

65 Ainsi Baltzer et al. 2011, p. 2597.

66 Je remercie Jan Rückl d'avoir attiré mon attention sur cette possibilité, qui est peut-être la plus vraisemblable.

67 Jer 27,39a LXX (= Jer 50,39a TM).

68 Le substantif est attesté pour la première fois dans la LXx. Il apparaît également en Sap 17,3, toujours avec la signification de « spectres », « hallucinations ». La reconstruction 
Tous ces exemples montrent la complexité du cadre interprétatif des habitants des ruines dans la LXX où, dans la plupart des cas, il est difficile d'identifier une logique de correspondance entre le grec et l'hébreu. Le manque de cohérence entre Is 13 et Is 34 est particulièrement frappant car le même catalogue d'animaux apparaît en hébreu et en grec mais les parallèles utilisés ne sont pas les mêmes dans les deux passages. On peut avancer l'hypothèse qu'il s'agisse d'un répertoire conçu et reconnu comme un groupe, dont chaque élément ne possèderait pourtant pas une identité spécifique aux yeux du traducteur, vraisemblablement à cause de la diversité et de la complexité déjà inhérentes à ces listes dans la langue de départ: certains des termes (comme ohhîm, qippôz, lilitit) sont des hapax et d'autres (comme șiyyîm) ne renvoient pas à un type spécifique de créature mais peuvent englober plusieurs référents tant animaliers qu'humains ou démoniaques. Les éléments de ce répertoire, presque toujours à la forme plurielle, sont donc perçus comme interchangeables entre eux et sont exploités par le traducteur avec une certaine liberté. Cette attitude est davantage propre au traducteur d'Isaïe. Son souci semble moins être celui de fournir des équivalences ponctuelles que de donner une impression générale du contenu des listes, comme le démontre, entre autres, le manque de correspondances entre le nombre d'éléments dans le texte hébreu et grec ${ }^{69}$. Néanmoins, des tendances similaires sont également identifiables, dans une certaine mesure, dans les traductions grecques de Sophonie et Jérémie. Le dénominateur commun des créatures qui apparaissent dans le dossier grec est donc exclusivement constitué par l'espace qu'ils occupent: un espace désormais ouvert à l'entrée du démoniaque, n'hésitant pas, notamment, à faire intervenir des figures diverses de la marginalité, animalières et autres.

\section{3}

\section{Sirènes, autruches et démons}

Lorsque l'on regarde le dossier des habitants des ruines dans la LXX, on reste surpris de voir apparaître à côté des démons des créatures caractérisées par une dimension mythologique prononcée, telles que des sirènes et des onocentaures. Une telle combinaison nous invite à nous poser la question des traits partagés par ces créatures, nous permettant de mettre en évidence les éléments les caractérisant comme habitants des ruines aux yeux du traducteur.

du terme dans une inscription funéraire du IV ${ }^{\mathrm{e}}$ siècle av. n. è. (SEG 30.264.1-2) n'est pas assurée : le cas est discuté en détail par Aitken 2014, p. 42.

69 Il s'agit d'une démarche typique de la traduction des listes dans la Lxx d'Isaïe : voir, à ce sujet, les remarques de Williamson 1993. 
Puisque l'introduction des sirènes parmi les habitantes des ruines constitue une crux de vieille date qui a interrogé à la fois biblistes et classicistes ${ }^{70}$, nous commencerons par celle-ci.

Dans la LXX, on recourt aux sirènes ( $\sigma \varepsilon 1 p \eta ̂ v \varepsilon \varsigma)$ pour traduire à la fois l'hébreu tannîm et bənôt ya ănâ. Comme nous l'avons vu, ces deux termes sont souvent associés dans les descriptions des ruines, même s'ils connaissent également un emploi plus large en tant que topoi de la lamentation de l'individu exclu de tout groupe social et humain, comme le montre ce passage du prophète Michée ${ }^{71}$ :

TM : «Aussi vais-je me lamenter et hurler. J'irai déchaussé et nu. J'entonnerai une lamentation à la manière des chacals (kattannîm), un chant de deuil, comme les autruches (kibnôt ya ănâ) ».

LXX : «Car (elle) ira se lamenter et gémir, elle ira déchaussée et nue,

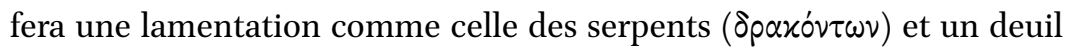

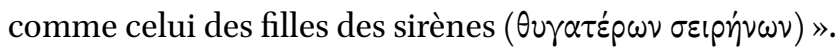

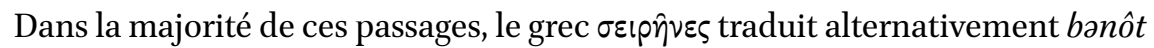
yaănâ et tannim, mais seulement quand ce dernier est associé aux bənôt ya ănâ (notamment en Is 13,21; 34,13; 43,20; Job 30,29), comme le montre le tableau suivant:

TABLEAU 3 Sirènes dans la LXX

Is 13,21

Is 34,13

Is 43,20

Jer 50,39 (= 27,39 LXX)

Job 30,29

Mich 1,8
שעירים

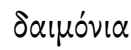

בנות יענה

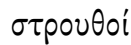

בנות יענה

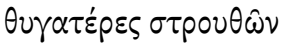

בנות יענה

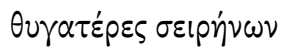

בנות יענה

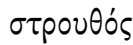

בנות יענה

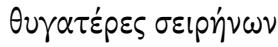

בנות יענה

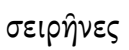

תנים

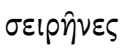

תנים

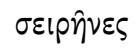

ציים

iv $\delta \alpha \lambda \mu \alpha \tau \alpha$

תנים

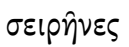

תנים

$\delta \rho \alpha x \omega \nu$

$70 \quad$ Voir, à cet égard, Kaupel 1935-1936 ; Antin 1961; Rahner 1964; plus récemment Pace 1998 et surtout Mancini 2012.

71 Mich 1,8. Sur ce thème voir Riede 2003. 
Lorsque c'est bənôt ya'ănâ qui est traduit par « sirène », tannîm peut à l'occasion être rendu par $\delta \rho \alpha \dot{x} \omega \omega \nu$, «serpent » / «dragon», comme en Mich 1,8. Cette équivalence se retrouve également dans la LXx de Jer 9,10. On peut émettre l'hypothèse qu'elle aurait été influencée par une proximité entre tannîm et tannîn, «monstre marin», «dragon», qui sont parfois déjà confondus en hébreu $^{72}$ : le serpent est d'ailleurs un animal qui convient très bien au paysage des ruines.

Lidentification des bənôt ya'ănâ est également plus problématique qu'on ne le pense. Traditionnellement, on estime que l'expression, toujours au pluriel, désigne des autruches, et cette forme est mise en relation avec l'hapax yāèn en Lam $4,3^{73}$ : «Même les tannîm offrent la poitrine et allaitent leurs petits; la fille de mon peuple est devenue cruelle comme les yəênîm dans le désert ». Toutefois cette identification est problématique dans la mesure où la forme est également attestée dans les listes d'oiseaux impurs de Lev 11 et Deut 14. Dans ce cas, les bənôt ya ănâ se trouvent dans une section de la liste occupée exclusivement par des rapaces et oiseaux de proie : la mention des autruches ici ne semble alors pas très logique ${ }^{74}$. En outre, il faut considérer que l'hébreu connaît au moins un autre nom pour l'autruche, à savoir *ronānâ, attesté au pluriel ranānîm seulement en Job 39,13-17. Le contexte de Job 39 ne laisse pas de doutes quant à l'identification avec l'autruche car il mentionne la croyance répandue dans l'antiquité selon laquelle l'animal abandonne ses œufs et ne s'occupe pas de ses petits. Lam 4,3 évoque également la cruauté des yəēnîm du désert envers leurs petits, ce qui renvoie à la même croyance, bien que l'animal soit nommé différemment.

La situation est ultérieurement compliquée par la présence d'un troisième lexème en sémitique pour «autruche», à savoir $n^{\prime} m / n$ r $a \bar{a} m \bar{a}$ ', attesté en différentes formes en hébreu postbiblique, en judéo-araméen, en syriaque et en $\operatorname{arabe}^{75}$. La relation entre ces trois zoonymes est loin d'être clarifiée et mériterait une étude supplémentaire : il est toutefois possible que l'expression bat ou bənôtya'ănâ indiquait, à l'origine, un petit rapace, puis qu'elle ait fait l'objet d'une resémantisation.

Il ne fait cependant pas de doute que les bənôt yaănâ étaient identifiées à des autruches dans les versions anciennes. Dans la moitié des occurrences citées dans le tableau, elles sont traduites littéralement en grec par $\sigma \tau$ povӨoí.

72 Voir, par exemple, Ez 29,3; 32,2. Pour une discussion sur la relation entre les deux lexèmes, voir Angelini 2018 a, p. 26-30.

73 Forme qere: le ketiv est incompréhensible.

74 Driver 1955 I, p. 12-16; Nihan 2017, p. 96-97.

75 SED II, no. 155, et Jastrow 1903, s.v. 
Par contre, nous avons vu que tannim était un terme beaucoup moins clair, compris dans ce contexte comme proche du champ sémantique des bənôt ya'ănâ. Le traducteur d'Isaïe avait certainement bien compris que ce couple ne faisait pas seulement référence à de simples exemplaires zoologiques, mais qu'il représentait également des figures par excellence de la marginalité géographique et physique, voire de la mort. De plus, si l'autruche était dans le Proche-Orient ancien un animal inquiétant sinon monstrueux, dans l'Antiquité classique elle était perçue comme un hybride hors catégorie et classée par Aristote parmi les $\alpha \dot{\mu} \varphi \circ \tau \varepsilon p i \zeta o v \tau \varepsilon s$, les animaux ambigus qui échappent à toute classification : avec des ailes énormes qui sont pourtant incapables de la faire voler, elle fonde la légendaire rapidité de sa course sur ses longues pattes; bipède, elle a les pieds fendus comme les quadrupèdes et son corps est couvert de plumes comme celui des oiseaux ${ }^{76}$. C'est peut-être son caractère d'hybride ailé maîtrisant les espaces marginaux, tels que les déserts et les steppes, qui a pu suggérer au traducteur l'emprunt aux sirènes de la tradition grecque. À cet égard, il n'est pas inutile de préciser que, en dépit de leur association mythique avec la mer, les sirènes ne sont pas des créatures marines ; leur habitat est plutôt celui des rochers, des îles, des écueils et des promontoires penchés sur les détroits et les bras de mer. En outre, la représentation de la sirène a également été employée par d'autres traducteurs, tels que ceux de Job et des petits prophètes, qui ont exploité d'autres nuances de l'imaginaire métaphorique associé aux tannim et aux bənôt ya ănâ, notamment la dimension sonore de la lamentation de l'individu sur sa mort sociale. Or, à l'époque hellénistique la sirène est surtout représentée dans l'art funéraire et son nom apparait dans la poésie funéraire comme terme de comparaison pour la lamentation funèbre, le défunt mort prématurément, voire le tombeau. Par exemple, l'une des rares épitaphes provenant de Karanis dans le Fayoum, datée à l'époque ptolémaïque, pleure la mort prématurée de la jeune fille Lysandros en la comparant à une sirène ${ }^{77}$.

Un passage du quatrième livre des Maccabées semble s'inscrire dans une atmosphère similaire puisque les cris de douleurs des jeunes juifs soumis à la torture et invoquant leur mère sont comparés au chant des sirènes et des cygnes: «Ni le chant des sirènes, ni la voix des cygnes ne provoquent autant d'affection en ceux qui écoutent, que la voix des enfants appelant leur mère

\footnotetext{
76 Arist., $P A 697 \mathrm{~b} 14-26$; GA 749b 17-25.

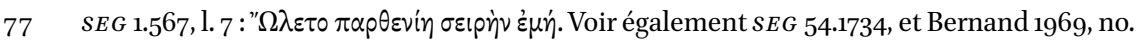
83 , p. 327-332 qui cite des exemples similaires d'époque romaine (ibid., p. 331). Étienne Bernand estime qu'il s'agissait d'une jeune musicienne car le relief, aujourd'hui détruit, représentait un protomé de femme avec une lyre à gauche et une cithare à droit. L'aspect musical et funéraire justifierait ainsi l'épithète de «sirène » attribuée à la jeune fille.
} 
aux milieux des supplices ${ }^{78}$. Il est donc vraisemblable que l'association à la lamentation funèbre justifie la traduction de tannîm et de bənôt ya'ănâ par

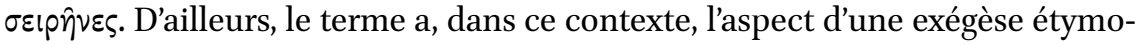
logique de bənôt ya ănnâ, littéralement « les filles du chant», à savoir, d'un point de vue grec, les sirènes. Il faut toutefois garder à l'esprit qu'ici nous n'avons pas à faire au chant séduisant, typique de la sirène homérique, qui charme l'auditeur par la promesse du savoir, mais plutôt à la lamentation chagrinée propre à la sirène funéraire. À cet égard, une hypothèse différente a été suggérée par Manolis Papoutsakis ${ }^{79}$, d'après qui la traduction de la Septante pourrait avoir été influencée par une tradition exégétique sur $\mathrm{Na} a m a h$, la sœur de Tubal-Cain, qui est implicitement comparée dans les Targums à une sirène, en tant que maîtresse de la musique et inventrice du chant. Papoutsakis se fonde, entre autres, sur le fait que le terme araméen utilisé pour traduire « autruche », nə'âmma', ressemble de très près au nom de la sœur de Tubal-Cain. Cette interprétation me paraît toutefois peu vraisemblable car, dans les passages bibliques concernés, l'accent n'est pas posé sur l'association avec le chant en général et les instruments de musique, mais plutôt sur la lamentation funèbre. En revanche, la construction targumique et rabbinique de $\mathrm{Na}$ amah comme « sirène » chanteuse et séduisante s'inspire - me semble-t-il - d'un imaginaire de la sirène qui se développe tardivement, en accord avec la représentation de cet hybride qu'on trouve dans le Physiologos et dans les commentaires des Pères.

Le couple sirènes/démons, qui apparaît pour la première fois dans la LXX d'Isaïe, jouira, effectivement, d'un certain succès dans la littérature des Pères de l'Église. Si le choix du référent mythique de la sirène fait par le traducteur introduit une dimension étrangère au texte hébreu, celle d'un mythe proprement «classique», cela a aussi des conséquences du point de vue de l'imaginaire grec. En effet la sirène n'était pas, à proprement parler, un démon dans la tradition hellénique, en tout cas jusqu'à une époque tardive et, notamment, chrétienne. Hybride mythologique, figure du passage et de la mort autant que de la séduction et du savoir, bien qu'elle puisse parfaitement correspondre à une notion contemporaine de démon, elle n'est jamais caractérisée comme telle par les sources anciennes à notre disposition. Cette définition, au moins en termes « émiques », n'était pas possible pour la sirène notamment car, surtout à l'époque hellénistique où l'on dispose de témoignages d'une démonologie grecque plus développée, les $\delta \alpha i ́ \mu o v \varepsilon \varsigma$ de la tradition littéraire et philosophique sont, pour la plupart, caractérisés par leur inconsistance et leur nature aériforme, c'est-à-dire par un manque de corps et non par l'hybridité du corps. Le

784 Mac 15,21.

79 Papoutsakis 2004. 
fait qu'une paire très similaire à celle de la LXx d'Isaïe se retrouve également en

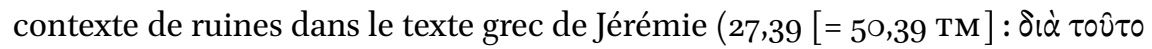

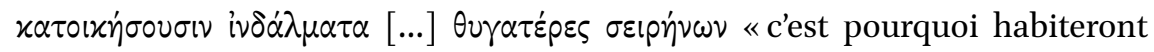
des spectres [...] et filles des sirènes »), dont le traducteur est d'habitude jugé comme moins créatif, est en ce sens révélateur. Il suggère que les démons sont à comprendre en ce contexte non comme des puissances néfastes, mais plutôt comme des apparitions liées à la sphère funéraire. Il s'agit d'ailleurs d'un domaine sémantique qui, comme on l'a vu, pouvait très bien être recouvert par $\delta \alpha i ́ \mu \omega \nu / \delta \alpha \mu o_{v}{ }^{\prime}$ v dans la tradition classique ${ }^{80}$.

Lassociation entre démons et sirènes a un certain succès dans les traditions juives d'époque hellénistique, non seulement en Égypte, mais également en Palestine. Elle est reprise, par exemple, au premier siècle de notre ère dans un passage de l'Apocalypse de Baruch, où le protagoniste, assis devant la porte du Temple après le départ de Jérémie, invite «les sirènes de la mer, les Lilithes du désert, les démons et les chacals des forêts » à élever tous ensemble un chant funèbre sur Sion ${ }^{81}$. Le texte, qui s'inspire notamment d'Is 34, réinterprète pourtant le passage isaïen par un mélange libre qui lui donne une véritable portée cosmique, associant les sirènes, les Lilithes et les chacals chacun à un espace géographique différent, et les mettant en parallèle avec les démons.

Les sirènes sont également évoquées dans le livre d'Hénoch, lorsqu'il est question de la punition des femmes qui se sont unies aux anges transgresseurs et seront, pour cette raison, transformées en sirènes ${ }^{82}$. Là encore, l'on pourrait se demander si, au lieu de la dimension sexuelle qui est parfois invoquée pour expliquer leur présence, ce ne sont pas plutôt l'aspect funéraire ainsi que leur nature mortelle qui devraient être soulignés dans ce cadre, étant donné que les Veilleurs sont également destinés à perdre leur immortalité à cause de la faute qu'ils ont commise ${ }^{83}$.

L'association entre sirènes, démons et onocentaures sera ensuite livrée au christianisme par le biais du Physiologos et des bestiaires médiévaux ${ }^{84}$. Ce lien entre sirènes et démons n'est d'ailleurs pas exclusif à la littérature apocryphe

8o $\quad$ Voir supra, $\S 2.2$.

813 Bar 10,8: «J'invoquerai les sirènes de la mer,/et vous, Lilithes (llyt'), accourez du désert,/ démons ( $\left.s^{\prime} d^{\prime}\right)$ et chacals, des forêts./Réveillez-vous et ceignez vos reins pour le deuil./ Entonnez avec moi des chants funèbres, avec moi gémissez » (traduction P. Bogaert, Sources Chrétiennes 144, Paris, Cerf 1969).

821 Hen 19,2: «Et les femmes de ces anges transgresseurs deviendront des sirènes » (trad. Silvestrini).

83 C'est l'hypothèse de Matteo Silvestrini, à paraître. La présence des sirènes ici pourrait avoir été suscitée par la mention des démons au verset précédent (1 Hen 19,1).

84 Voir, à ce sujet, Leclerq-Marx 2005 ; Angelini 2014. 
et aux commentaires chrétiens: il devient également plus évident dans la tradition grecque et romaine d'époque tardive où la sirène est rapprochée de toute une série de monstres féminins et nocturnes à l'apparence mi-humaine mi-animale, comme Lamia, Empousa ou Mormo, qui attaquent les hommes en les dévorant ou en les séduisant, et qui enlèvent les enfants à leurs mères. Un relief d'époque impériale provenant de Gaule atteste de cette évolution de l'imaginaire démoniaque associé aux sirènes : il montre un homme endormi en train d'être sexuellement attaqué par une femme ailée aux pattes d'oiseaux, laquelle agit de la même manière de ce qu'on appelle un démon « incube ${ }^{85}$. C'est en puisant dans cette tradition que Jérôme identifiera Lilith à une Lamia dans la Vulgate, un choix qui avait déjà été opéré par Symmaque ${ }^{86}$. À cet égard, il est bien compréhensible que les réviseurs de la Lxx, Aquila, Symmaque et Théodotion, refusent à l'unanimité l'emploi des sirènes en Is 13,21 où il est dit «les bənôt ya ănâ s'établiront là-bas», ainsi que dans un contexte très similaire en Is 34,13, en suggérant plutôt une traduction littérale de bənôt

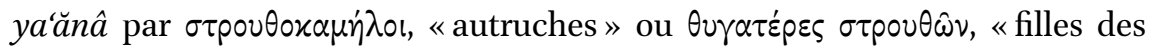

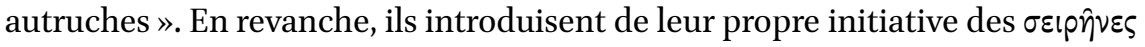

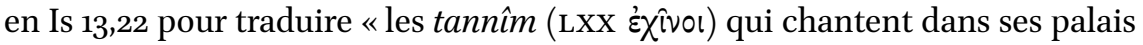
d'agrément», en les considérant sans doute plus appropriées dans ce contexte où l'on fait mention du chant et du «plaisir » ('ōneg), évidemment interprété comme plaisir sexuel. Cette option est aussi adoptée par Jérôme en Is 13,22, qui parle des sirenae in delubris voluptatis ( « sirènes dans les temples du plaisir »): il s'agit de la seule fois où Jérôme garde la mention des sirènes parmi les six attestations qu'il a repérées dans la $\mathrm{LXX}^{87}$.

\section{$4 \quad$ Isaï 34 et les onocentaures : vers un nouveau paradigme épistémologique}

La dernière des créatures qui va occuper notre attention est finalement l'onocentaure, animal qui intervient à plusieurs reprises parmi les habitants des ruines dans la LXX d'Isaïe (set exclusivement ici), d'abord en Is 13,22 :

\footnotetext{
85 Boston, Museum of Fine arts, RES 08. 34c. Sur l'interprétation de ce relief, voir Harris 2009, p. 35 .

86 Is 34,14 (Vulgate) : et occurrent daemonia onocentauris et pilosus clamabit alter ad alterum ibi cubavit lamia et invenit sibi requiem. Voir aussi Jer., Comm. in Isaiam 10,34, et Ziegler 1939, p. 245-246, apparatus II.

87 Voir, à ce sujet, Antin 1961; Pace 1998.
} 
וענה איים באלמנותיו

ותנים בהיכלי ענג

וקרוב לבוא עתה בהיכלי ענג

וימיה לא ימשכו עתה

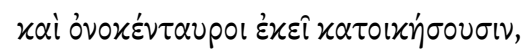

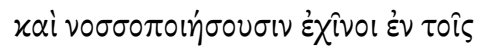
olixols $\alpha \dot{\tau} \tau \hat{\omega} v$.

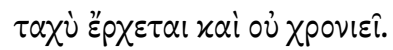

Et les onocentaures y habiteront, les hérissons feront leur nid dans leurs maisons :

(cela) arrivera rapidement, et ne sera pas retardé.

Ensuite des onocentaures sont nommés à trois reprises en Is 34,11 et 14:

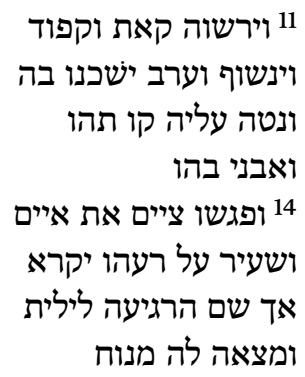

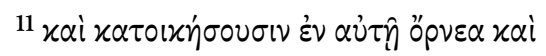
ह’ $\chi \hat{\imath}$ ol

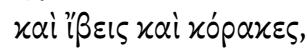

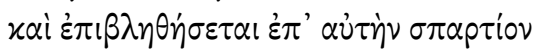

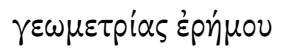

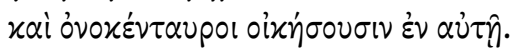

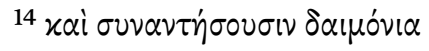

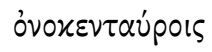

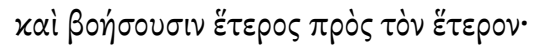

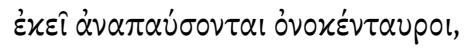

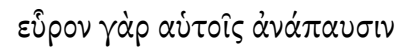

${ }^{11}$ mais y habiteront les oiseaux et les hérissons, les ibis et les corbeaux ; la mesure de la désolation sera jetée sur elle et les onocentaures habiteront là-bas. ${ }^{14}$ Et les démons se rencontreront avec les onocentaures et s'appelleront l'un l'autre à grands cris ;

là-bas se reposeront les onocentaures, car ils trouveront leur repos.

L'onocentaure doit notamment son apparition sur la scène de la littérature grecque à la plume du traducteur d'Isaïe : il s'agit, en effet, du premier témoignage de cet hybride qui constitue une variante mineure du centaure, à savoir une créature dont la partie postérieure est celle d'un âne et la partie antérieure celle d'un être humain. La compilation encyclopédique sur la «personnalité 
des animaux » de Claude Élien, qui travaillait à Rome au deuxième siècle de notre ère, fournit une description détaillée de l'onocentaure. Élien cite, parmi ses sources sur l'onocentaure, Cratète de Pergame, philosophe stoïcien et directeur de la bibliothèque de Pergame, lequel fait à son tour remonter ses informations à un certain Pythagore, qui était géographe et stratège à la cour de Ptolémée Philadelphe ${ }^{88}$. Sur ce point il faut justement rappeler que les cours lagides étaient caractérisées par un intérêt particulier pour la faune exotique et sauvage dont ils faisaient collection et que à l'occurrence pouvaient exposer au public en signe de prestige, comme dans le cas du fameux cortège des Ptolémaia, fête instituée par le Philadelphe en l'honneur de son père défunt. Plusieurs sources anciennes témoignent en outre des efforts faits par le souverain pour se procurer des exemplaires divers d'animaux rares ${ }^{89}$. Quant à l'onocentaure plus spécifiquement, son seul témoignage iconographique assuré provient d'une mosaïque romaine dite mosaïque du Nil ou mosaïque Barberini, qui ornait le nymphée de l'ancien sanctuaire de Préneste et qui remonte au premier siècle av. n.è. La mosaïque, d'origine alexandrine, combine un riche paysage nilotique sur la partie inférieure, composé de bateaux, bâtiments et hommes avec un territoire sauvage et montagnard sur la partie supérieure, qui représente le confin au sud de l'Égypte, à savoir l'Éthiopie. Comme l'observe Jean Trinquier ${ }^{90}$, l'Éthiopie est ici représentée comme une terre de frontière, pour ainsi dire opposée à la civilisation égyptienne figurée dans la partie supérieure : la partie éthiopienne est en effet déserte, dépeuplée de villes et d'êtres humains, remplie de végétation et d'animaux bizarres. Parmi les curieux animaux qui décorent ce paysage, on reconnait par l'inscription

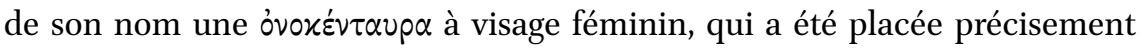
sur la frontière entre l'Égypte et l'Éthiopie. En s'appuyant sur ces témoignages, on peut donc conclure que des croyances relatives à cette créature étaient vraisemblablement en circulation à l'époque hellénistique et qu'elles étaient connues de notre traducteur, mais de manière imprécise, car l'onocentaure d'Élien et de la mosaïque nilotique sont de genre féminin, alors que l'onocentaure biblique est masculin ${ }^{91}$. Cet hybride était caractérisé par un arrière-plan mythologique important, celui des centaures, figures grecques de la liminalité

88 Ael., NA 17, 8. Voir Fraser 1972, p. 306, note 365. Pour une synthèse des sources anciennes sur l'onocentaure, voir Preisendanz 1939.

89 Sur tous ces aspects, voir l'étude exahustive de Trinquier 2000.

90 Trinquier 2004, p. 46-53.

91 Sur cette mosaïque, voir Meyboom 1995, p. 20-22, 43-50, 111-114, Trinquier 2004. Sur la correspondance entre la scène de la mosaïque et le témoignage d'Elien, voir Schettino 2005, p. 288-289. Voir également Aitken 2014, p. 77-79. 
et du renversement ${ }^{92}$, tout en étant en même temps un animal du scénario ethnographique égyptien. En même temps en contexte alexandrin il était «bon à penser » l'altérité et l'exotisme, comme le démontre le fait que dans la mosaïque il est un marqueur des frontières éthiopiennes. Son nom contenait de plus une référence à l'âne, en l'occurrence certainement l'âne sauvage, au vu de sa localisation dans les steppes et les déserts. Or, lâne sauvage, ou onagre, est représenté dans les sources à la fois bibliques et classiques comme fier et inapprivoisable, voire incontrôlable. Il fait, en outre, partie des animaux qui peuvent apparaître dans les ruines, comme nous l'avons vu dans le passage d'Is 32,14, et il est déjà nommé en ce sens dans les traditions proche-orientales plus anciennes (par exemple dans l'inscription d'Assurbanipal). L'onocentaure remplit, donc, toutes les conditions pour rentrer dans le catalogue des habitants des ruines dont l'auteur avait compris le potentiel «para-naturel », même s'il n'arrivait plus à identifier avec précision certains référents tels que Lilith ou les 'iyyîm. À cet égard, puisque le terme traduit deux fois 'iyyîm (en Is 13,22;34,14), l'on pourrait même envisager l'hypothèse, comme certains commentateurs le suggèrent, quà l'origine de ce choix le traducteur ait opéré une identification avec un quadrupède des landes isolées, tel que, par exemple, le chacal, auquel il aurait donné une touche «égyptianisante » et qu'il aurait ensuite élargi à d'autres passages du texte ${ }^{93}$.

La présence de l'onocentaure parmi les animaux des ruines nous offre une clé fondamentale pour interpréter les changements qui ont eu lieu dans la représentation des animaux des ruines de la LXX d'Isaïe, notamment si on les considère en relation avec leur contexte de référence, c'est-à-dire la culture grecque de l'Égypte hellénisée, que le traducteur d'Isaïe semble bien connaître. Dans l'ensemble des passages décrivant les habitants des ruines, les sirènes, les démons et les onocentaures, bien que relevant d'un arrière-plan mythologique, sont soumis à un processus de naturalisation progressive qui les amène à coexister avec les bêtes sauvages, les hérissons, les ibis, les cerfs, et d'autres animaux de ce type, sans que leur inclusion dans ces catalogues ne semble soulever de problèmes particuliers. De plus, dans la version grecque d'Isaïe, et notamment en Is 34, l'aspect démoniaque des habitants des ruines est intégré à un paysage qui est présenté comme définitivement pacifié ou pacifique. Cette impression est corroborée par la mention des cerfs vers la fin de

92 Voir, à ce sujet, entre autres, Bremmer 2012 et bibliographie relative.

93 On pourrait également se demander si le choix du préfixe ono-, au lieu d' hippo-, plus commun, ne constitue pas une référence voilée à Seth, dieu égyptien du chaos et des steppes, à la tête d'âne ou de canidé. Sur l'animal séthien, voir récemment Mathieu 2011. 
la péricope, au lieu des vautours originaux en Is 34,15 b, qui se rencontrent en toute tranquillité :

TM : « Là encore, se rassembleront des vautours (dayyôt), chaque femelle avec son compagnon ».

LXX : « Là-bas des biches ( $\left(z^{\prime} \lambda \alpha \varphi \iota\right)$ se sont rencontrées, et elles ont regardé l'une le visage de l'autre».

Le remplacement des vautours par des biches est à inscrire à l'intérieur d'un changement plus profond par rapport à l'hébreu dans la traduction des versets $15^{-17}$, où le territoire n'est plus laissé sous la possession des habitants des ruines, mais sera restitué aux hommes (Is 13,17b Lxx : «Ils seront votre héritage pour l'éternité »). Ce changement s'explique par une exégèse qui concerne l'oracle dans son entier. Comme je l'ai démontré ailleurs, dans la LXX d'Is 34,817 le jugement divin est exercé non sur Édom, comme c'est le cas en hébreu, mais sur Sion, qui ne fait donc pas l'objet d'une condamnation définitive; le texte grec prépare ainsi le passage vers le rassemblement à Sion qui est décrit en Is $35^{94}$.

D'un point de vue plus général, la coexistence d'animaux et de créatures de différents genres et espèces représentée dans la LXX d'Isaïe, et surtout en Is 34, s'inscrit parfaitement dans l'horizon culturel hellénistique. Le monde hellénistique représente en effet un univers dont les frontières s'élargissent progressivement et dont les ह̇ $\sigma \alpha \alpha \tau \alpha$ í, les périphéries, font de plus en plus l'objet d'une vraie connaissance et suscitent un intérêt continuellement renouvelé, notamment à cause de tous les êtres merveilleux qu'elles sont censées générer. Par conséquent, les frontières du savoir naturel et zoologique deviennent, surtout dans l'hellénisme tardif, moins rigides, au point d'inclure des cas qui, jusqu’à Aristote, auraient été très problématiques sinon franchement impossibles à intégrer, comme les hybrides du mythe. Ce n'est pas un hasard si, au tournant de l'ère, on observe une production intense de catalogues et de recueils de merveilles et de paradoxographies. Pline l'Ancien, par exemple, bien qu'il se présente comme héritier d'Aristote (c'est-à-dire qu'il se situe lui-même du côté de la science) affirme dans son Histoire Naturelle avoir vu de ses yeux toute une série d'êtres extraordinaires parmi lesquels figurent des Tritons, des Néréides, des hommes marins (mi-hommes mi-poissons) et notamment

94 Voir notamment le texte grec d'Is 34,8: «Car c'est le jour du jugement du Seigneur, une année de rétribution, de jugement de Sion ». Pour une analyse complète de la relocalisation de l'oracle d'Édom à Jérusalem dans la LXX d'Is 34, voir Angelini 2016. 
un hippocentaure amené d'Égypte à la cour de l'empereur Claude, conservé dans le miel ${ }^{95}$. Élien utilise notamment la description de l'onocentaure comme preuve définitive de l'existence des centaures, dont la vraisemblance avait fait

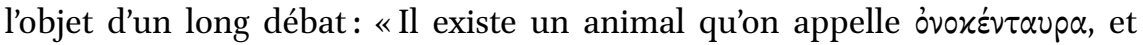
quiconque l'a vu ne saurait douter que la race des centaures a existé et que les artistes n'ont pas falsifié la nature, mais que le temps a produit ces animaux en combinant des corps dissemblables pour en faire un seul ${ }^{96}$.

Cette orientation épistémologique a, entre autres, des conséquences qui touchent à la notion même du démoniaque, notion qui se trouve dans une certaine mesure privée de son caractère menaçant et de son lien original avec la sphère du divin, pour se rapprocher de celle du merveilleux et du prodigieux au sens large. À cet égard, on constate dans la littérature de l'époque hellénistique la mise en place de deux tendances distinctes. D'un côté, se développe dans la philosophie un savoir anthropologique et cosmologique sur les démons qui leur assigne un espace d'habitation, des attitudes comportementales et des compétences spécifiques ${ }^{97}$, les classifiant, ainsi, à l'intérieur de la taxinomie du monde naturel. De l'autre côté, dans la langue courante, l'adjec-

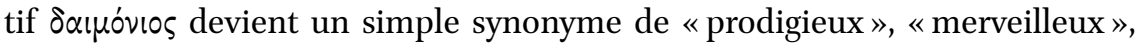
«qui suscite la stupeur »; il est, entre autres, employé avec cette nuance dans l'œuvre de Claude Élien ${ }^{98}$. Les animaux, les monstres et les démons du passé mythologique deviennent ainsi les mirabilia du présent ethnographique des régions inconnues et périphériques du monde. La représentation des habitants des ruines de la LXX d'Isaïe, qui correspondent dans la logique du texte

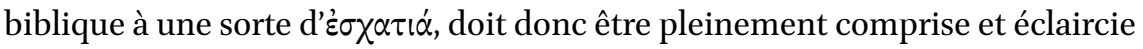
à la lumière de ce nouveau contexte culturel, contexte dans lequel l'Alexandrie lagide jouait d'ailleurs un rôle de premier rang. À ce propos, et de manière plus générale, je souhaite attirer l'attention non seulement sur l'importance de la prise en compte du contexte hellénistique pour la compréhension du travail des traducteurs, démarche sur laquelle la recherche récente a, certes, raison d'insister, mais également sur un aspect qui demeure jusqu'à présent moins reconnu, à savoir la valeur de la LXx pour la reconstruction dudit contexte. La LXX représente à ce titre un dossier remarquable dans la mesure où il est considérablement ancien, car il précède les descriptions des auteurs les plus étudiés pour la reconstruction des paradigmes épistémologiques hellénistiques tels

95 Plin., $N H$ 7, 35. Sur le cadre épistémologique des auteurs de l'hellénisme tardif, voir les études fondamentales de Romano 1998 et Li Causi 2003.

96 Ael., NA 17, 9 (trad. Zucker, Paris, Les Belles Lettres, 2002).

97 Voir p. 66-67.

98 Ael., NA 17, 9. 
que Diodore de Sicile, Strabon, Pline, Élien et autres. Alors que le thème des mirabilia a reçu l'attention de plusieurs spécialistes de l'ethnographie hellénistique, la contribution de la LXx à la compréhension et au développement d'un tel paradigme a été jusqu’à présent négligée. Elle mérite en revanche d’être été relevée et exploitée. Bien que la recherche menée dans ces pages se soit focalisée surtout sur le corpus prophétique et notamment sur la Lxx d'Isaie, il y a des bonnes raisons d'envisager que d'autres corpus, tels que par exemple le Pentateuque, le livre de Job, de Proverbes et d'autres encore, représentent en ce sens un domaine de recherche fécond, qui reste encore à explorer.

En conclusion, plusieurs éléments ressortent de ce parcours parmi les habitants des ruines dans la LXx. Concernant les techniques de traduction, l'analyse des versions grecques a mis en évidence un manque de correspondance et de systématisme dans le choix des noms employés. Cette donnée serait plutôt à attribuer à l'attitude spécifique de la traduction de la Septante d'Isaïe; néanmoins, étant donné qu'on la repère en partie dans la LXX de Sophonie et de Jérémie, elle relèverait également de la nature composite de ce bestiaire, à l'intérieur duquel il apparaît difficile pour les traducteurs de détecter des individualités précises. À propos de la fonction générale de l'espace des ruines, et au vu de la relocalisation de l'oracle d'Is 34 d'Édom à Sion, on peut, en outre, constater qu'il n'y a plus d'écart significatif entre la ruine annoncée aux nations et celle qui atteindra Israël : le paysage est exactement le même à Babylone et à Jérusalem, même si le destin final des deux villes demeure différent.

En ce qui concerne les isotopies du texte hébreu, on relève qu'elles ont toutes été bien préservées par les traducteurs : la dimension sonore du paysage des ruines, le rapport des habitants des ruines à la mort, ainsi que la nature hétérogène et ouverte du bestiaire sont bien présents dans le texte grec. Quant à l'hétérogénéité du bestiaire, deux phénomènes sont à mettre en évidence. Premièrement (1), le caractère démoniaque qui demeurait implicite dans le texte source fait l'objet d'une interprétation qui le rend clair et explicite, comme le démontre l'emploi du substantif $\delta \alpha \mu o ́ v ı \alpha$, introduit pour traduire différents noms hébreux. De ce point de vue, la Septante garde une forte continuité avec les traditions orientales qui prévoyaient toujours des démons parmi les habitants des ruines, tout en se plaçant à l'origine d'une nouvelle tradition exégétique qui reliera plus étroitement entre eux les Lilithes, les sirènes et les onocentaures. Ce rapport dynamique entre la Lxx et des traditions proche-orientales anciennes n'a pas été, me paraît-il, mis en valeur jusque-là : il s'agit néanmoins d'un aspect important du point de vue historico-religieux, sur lequel je réfléchirai à nouveau dans les conclusions de mon enquête.

La tendance exégétique qui explicite le potentiel démoniaque de la Bible hébraïque est propre surtout à la Lxx d'Isaïe; elle est néanmoins en accord 
avec d'autres développements de l'époque du Second Temple, tels qu'ils sont attestés par exemple à Qumrân. J'ai déjà évoqué les «Chants du Sage », des compositions destinées à chasser les mauvais esprits qui ont dû, probablement, être écrites par un sage de la communauté, nommé le « Maškil » et qui ont été conservées sur deux rouleaux, respectivement $4 \mathrm{Q}_{510}$ et $4 \mathrm{Q} 511$. Le premier fragment de 4Q51o (le mieux préservé parmi les deux manuscrits) établit la liste d'une série de créatures qui sont à exorciser, dans la séquence suivante ${ }^{99}$ :

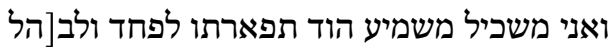

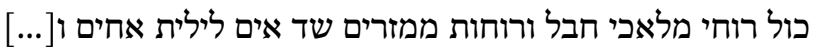 והפוגעים פתע פתאום לתעות רוח בינה ממרזים
}

\author{
Et moi, je suis un sage, qui proclame la majesté de Sa splendeur, pour \\ effrayer et ter[rifier] \\ tous les esprits d'anges de corruption et les esprits des bâtards : démon, \\ hurleurs, Lilith, hiboux ('ọhim) et [...] \\ ceux qui frappent à l'improviste pour l'égarement de l'esprit \\ d'intelligence.
}

Même si le texte est en partie mutilé, Eibert Tigchelaar, Noam Mizrahi et Hector Patmore ${ }^{100}$ ont récemment montré que ce passage contient vraisemblablement une série de créatures très similaire à celle qui apparaît dans les passages d'Is 34,14 et 13,2. Nous avons, donc, ici affaire à un phénomène parallèle de démonisation explicite des habitants des ruines que l'on peut comparer avec la LXX d'Isaïe. Alors que, dans le cas du Chant du Mashkil, cette démonisation est réalisée en ayant recours à des notions proprement qumrâniennes, comme, par exemple, celle des « esprits des bâtards » et des « anges de corruptions », le traducteur d'Isaïe réinterprète les habitants des ruines selon des catégories qui sont plus proches du monde grec et notamment gréco-égyptien.

Deuxièmement (2), la transposition de l'imaginaire hébreu en catégories grecques, ou plus précisément hellénistiques (comme le montre par exemple le choix de l'onocentaure, marqueur de l'Égypte hellénisée), est filtrée par le

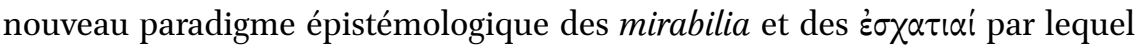

99 4Q510 fr. 1, l.4-6 (trad. Baillet 1982, DJD 7, p. 216, modifiée en suivant les remarques de Tigchelaar 2018; Mizrahi et Patmore 2019).

100 Tigchelaar 2018, p. 128, note 13; Mizrahi et Patmore 2019. Ces chercheurs ont démontré que les formes šd et 'ym, séparées par un espace dans le fragment, ne sont pas à lire comme un pluriel irrégulier de šêd, comme le proposait Baillet (1982), mais sont effectivement deux noms distincts : šêd, « démon », et 'ym étant une forme équivalente des 'iyyîm bibliques. 
le traducteur grec a été influencé. Cela implique, dans la perspective du traducteur et de l'auditeur de l'époque, un certain degré de naturalisation du scé-

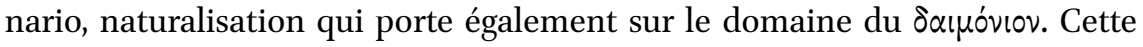
dimension est particulièrement évidente dans le texte grec d'Isaïe en raison, non seulement, de la nature littéraire de cette traduction, mais aussi car il s'agit du texte qui présentait le caractère démoniaque le plus marqué déjà dans le texte de départ parmi ceux qui composent le dossier des habitants des ruines. Par conséquent, il me paraît possible de conclure que, dans le contexte des habitants des ruines de la LXx, les démons dégagent une double isotopie: en tant qu'apparitions spectrales et en combinaison avec les sirènes, ils sont liés au domaine de la mort et au registre funèbre; en même temps, et tant qu'appartenant à l'horizon naturaliste des ह̇ $\sigma \chi \alpha \tau \iota \alpha$, ils renvoient aux merveilles propres aux espaces périphériques et fantastiques. Ces deux aspects de la représentation du démoniaque ne doivent pas nécessairement être compris comme étant en contradiction entre eux : ils sont plutôt témoins de la richesse de cette notion, ainsi que de la capacité des traducteurs grecs à l'exploiter en fonction de leur contexte. 